\title{
A NON-MINIMAL SUPERGRAVITY MODEL CONSISTENT WITH ALL EXPERIMENTAL CONSTRAINTS*
}

\author{
M. QUIRÓS ${ }^{\dagger}$ and G.L. KANE \\ Randall Laboratory of Physics, University of Michigan, Ann Arbor, MI 48109, USA
}

H.E. HABER

Department of Physics, University of California, Santa Cruz, CA 95064, USA

Received 23 October 1985

\begin{abstract}
We examine non-minimal supergravity models by omitting some of the usual simplifying assumptions. In particular, the $\mathrm{SU}(2) \times \mathrm{U}(1)$ breaking is uncoupled from the higgsino mass parameter, and the photino to gluino mass ratio is not rigidly fixed. We construct and examine in detail an interesting, and perhaps phenomenologically relevant model, with heavy squarks and light gluinos; the lightest supersymmetric particle is a higgsino $\tilde{\mathrm{h}}$, so the photino decays $\tilde{\gamma} \rightarrow \gamma \tilde{\mathrm{h}}$. We discuss ways to determine all of the parameters of the model, and its experimental implications. One interesting consequence is a new way to produce Higgs bosons, $e^{+} e^{-} \rightarrow Z^{0} \rightarrow \tilde{h} \tilde{Z} \rightarrow$ $\tilde{\mathrm{h}} \tilde{\mathrm{h}} \mathrm{H}^{0}$, with a rate that depends on the $\tilde{\mathrm{Z}}$ mass but could be as large as almost one percent of all $Z^{0}$ decays even for $m_{\mathrm{H}}$ up to about $\frac{2}{3}$ of $M_{\mathrm{Z}}$; the signature is $\mathrm{H}^{0}+$ missing energy, which is quite good for detection.
\end{abstract}

\section{Introduction}

During the last few years, a great deal of effort has been devoted to studying grand unified theories coupled to $N=1$ supergravity and their low-energy predictions [1]. In particular it was proved that spontaneously broken $N=1$ supergravity induces a relic soft breaking of $N=1$ supersymmetry [2] which can trigger radiative breaking of the electroweak theory [3-8], and be consistent with the recently reported top-quark mass [9], $m_{t}=(40 \pm 10) \mathrm{GeV}$. The entire supersymmetric spectrum depends on four parameters (once we have fixed $m_{\mathrm{t}}$ and the gauge couplings): $m_{3 / 2}$ (the gravitino mass), $m_{\bar{v}}$ (a common Majorana gaugino mass at $M_{\mathrm{X}}$ ), $m_{4}$ (a supersymmetric higgsino mass) and $A$ (a parameter [1] related to the super-Higgs mechanism). The condition for $\mathrm{SU}(2) \times \mathrm{U}(1)$ breaking determines $m_{4}$ so that we are left with three free parameters: $m_{3 / 2}, m_{\tilde{v}}$ and $A$.

On the other hand, much effort has also been given to proposing and finding possible experimental signatures of supersymmetric particles [10]. Supersymmetric

\footnotetext{
* Research supported in part by the US Department of Energy.

' Permanent address: Instituto de Estructura de la Materia, Serrano 119, Madrid-6, Spain.
} 
theories usually have a conserved discrete symmetry, $R$-parity, which requires supersymmetric particles to be pair produced and the lightest supersymmetric particle (LSP) to be absolutely stable. The LSP interacts very weakly with matter, so the cleanest signal for supersymmetry is missing energy. Thus the large transverse energy mono-jets with large missing transverse momentum at the CERN $\bar{p} \bar{p}$ collider reported [11] by the UA1 collaboration are the types of events expected from supersymmetry.

In early 1984, the analysis of data from the 1983 run at the CERN $\bar{p}$ p Collider (at $\sqrt{s}=540 \mathrm{GeV}$ ) by the UA1 collaboration resulted in the report of monojets which seemed to be unexplainable by the standard model [11]. In the fall of 1984, more data were taken at a slightly higher energy, $\sqrt{s}=630 \mathrm{GeV}$. More than twice the luminosity (as compared to the 1983 run) was collected. It seems clear from the recent report on the 1984 data [12] that the missing-energy events which are seen are (for the most part) less dramatic and possibly explained by standard model backgrounds.

Recently, a general study of all supersymmetric processes giving rise to missing energy events, subject to UA1 1984 running conditions, has been performed by Barnett et al. [13]. Under the assumption that the photino is the LSP, Barnett et al. find that scalar-quarks and gluinos must be quite heavy (whether the UA1 data contains an anomalous missing momentum signal or not): $M_{\tilde{\mathrm{q}}} \geq 65-75 \mathrm{GeV}$ and $M_{\tilde{\mathrm{g}}} \geq 60-70 \mathrm{GeV}$ (similar conclusions have been obtained in ref. 14). On the other hand, if the higgsino is the LSP, these limits are substantially weakened. In this case, when scalar-quarks and/or gluinos are produced, they decay into photinos which subsequently decay into higgsinos. The missing-energy distribution is therefore softened, and fewer events pass the UA1 cuts and triggers. Barnett et al. estimate that the new limits are $M_{\tilde{\mathrm{q}}} \geq 45-60 \mathrm{GeV}$ and $M_{\tilde{\mathrm{g}}} \geq 40 \mathrm{GeV}$, although they argue that light gluinos $\left(M_{\tilde{\mathrm{g}}} \leq 5 \mathrm{GeV}\right)$ cannot be ruled out. The reason $[15,13]$ for this is that the gluino fragmentation further softens the photino energy spectrum; this effect becomes more pronounced as the gluino mass is reduced. Thus, for light gluinos, not enough events pass the UAl cuts and triggers for this scenario to be excluded if the higgsino is the LSP.

Using the above results for motivation, we have studied the possibility of constructing low-energy supergravity models with light gluinos, heavy squarks [16] and the higgsino as the LSP. We require that these models satisfy constraints from cosmology so that higgsinos which have survived since the big bang do not lead to too much mass in the universe. In the case of a pure higgsino, no relation exists between the higgsino and gluino mass. Thus, a higgsino $\tilde{h}$, with a mass $M_{\tilde{h}} \leqq \mathrm{O}(100)$ $\mathrm{eV}$, is from a cosmological point of view similar to light massive neutrino and is therefore acceptable.

This possibility is, however, difficult to reconcile with $S U(2) \times U(1)$ radiative breaking in the usual low-energy supergravity for the following reason. By studying the neutralino mass matrix we find that, for the experimentally allowed range of the 
top quark mass, $m_{\tilde{h}}=m_{4}$. However, $m_{4}$ is related to the parameter $m_{3}^{2}$ which governs the electroweak breaking scale by the relation

$$
m_{3}^{2}=B m_{3 / 2} m_{4}
$$

where $B=A-1$ at $M_{\mathrm{X}}$ is a number of order unity. Relation (1.1) implies the radiative breaking condition $m_{4}=\mathrm{O}\left(m_{3 / 2}\right)$ which is incompatible with such a light higgsino. This time even the solution of electroweak breaking triggered by the heavy "up" quark of a fourth generation is unacceptable; in that case the supersymmetric spectrum would contain an axion with a mass around $1 \mathrm{MeV}$, in conflict with particle physics experiments.

The essential problem here is that the same parameter $m_{4}$ governs the higgsino mass and electroweak breaking, via the relation (1.1). The way out is to construct a model where the higgsino mass and the electroweak breaking scale get decoupled; i.e. a model where $m_{4}$ and $m_{3}^{2}$ are two independent parameters. There is nothing against this possibility from the point of view of a softly broken supersymmetric theory. In fact the renormalization group equation of $m_{3}^{2}$ is [3]

$$
\begin{aligned}
\mu \frac{\mathrm{d} m_{3}^{2}}{\mathrm{~d} \mu}=\frac{1}{(4 \pi)^{2}}\{ & \left(-3 g_{2}^{2}-g_{1}^{2}+3\left|h_{\mathrm{t}}\right|^{2}\right) m_{3}^{2} \\
& \left.-\left(6 g_{2}^{2} M_{2}+2 g_{1}^{2} M_{1}-6 A\left|h_{\mathrm{t}}\right|^{2} m_{3 / 2}\right) m_{4}\right\},
\end{aligned}
$$

where $M_{i}=\alpha_{i} / \alpha_{\mathrm{GUT}} m_{\hat{\mathrm{v}}}$. If $m_{4} \neq 0$, then even if $m_{3}^{2}=0$ at $M_{\mathrm{X}}$, a non-zero value for $m_{3}$ is generated at lower scales by the renormalization group equation. However if $m_{4}=0$ the equation for $m_{3}^{2}$ is homogeneous (although it is a soft breaking parameter) and has a fixed point at zero. In that case, if eq. (1.1) holds, then $m_{3}^{2}=0$ at all scales; but if eq. (1.1) does not hold, even if $m_{4}=0, m_{3}^{2} \neq 0$ at any scale provided that $m_{3}^{2}\left(M_{\mathrm{X}}\right) \neq 0$.

It is usually argued that the decoupling of the $A$ and $B$ parameters (which is to some extent related to the decoupling of the $m_{4}$ and $m_{3}^{2}$ parameters) can be accomplished in a general $N=1$ supergravity theory with non-canonical kinetic terms by complicating sufficiently the Kähler potential. (We show an alternative way to do this.) The advantages of such a situation have been recently stressed in ref. [20]. It was shown that by keeping the relation $B=A-1$ and trying to fit supersymmetric spectra to the possible supersymmetric mechanisms for monojet production, only very restrictive values of the $A$ parameters (for instance $2.95<A<3$ or some negative values) are found [20,21], but by letting $A$ and $B$ behave as free parameters all restrictions on $A$ disappear. However in the only well-known $N=1$ supergravity theory with non-canonical kinetic terms, the no-scale models [22], the relation $A=B$ leads to similar constraints. On the other hand the flat limit, and the 
separation between hidden and observable sectors, is not so well defined in general non-minimal supergravity as it is in minimal supergravity, so that to postulate the existence of such a non-minimal model may be risky.

Looking for a model based on $N=1$ supergravity with canonical kinetic terms and where $m_{3}^{2}$ and $m_{4}$ get decoupled may seem, at first sight, a complicated task. However the low-energy theory depends on the particular super-Higgs mechanism we choose in the hidden sector. Actually two years ago Soni and Weldon [23] studied the most general super-Higgs mechanism consistent with the flat limit of the theory and showed that, even in the case of canonical kinetic terms, the low-energy theory had much more freedom than was usually thought. Since in the low-energy theory the parameters depending on the super-Higgs mechanism are usually considered as free parameters, or boundary conditions, there is no reason to consider only the low-energy theory coming from a particular super-Higgs mechanism. On the contrary, only after the most general super-Higgs mechanism has been used in the hidden sector of the theory are we allowed to consider the soft-breaking parameters, which appear in the low-energy limit of the observable theory, as free parameters or boundary conditions. We have found that, in the standard supersymmetric model, the low-energy effective theory depends on five parameters: $m_{3 / 2}$, $m_{\bar{v}}, m_{4}, A$ and $m_{3}^{2}$. Only in some special cases does eq. (1.1) hold and then one gets the usual result.

The super-Higgs mechanism leading to the most general low-energy theory is shown in appendix A. It is an adaptation of the Soni and Weldon mechanism and we have included it for completeness. In addition, we discuss the low-energy theory in the fermion sector in this more general case.

To put it somewhat differently, we do believe it is important to construct theoretical models to guide thinking and analysis of experimental data. Interestingly, attempts to interpret the reported monojet data in terms of $N=1$ broken supergravity models appear likely to fail in the case of the minimal softly broken theory. We do not believe that the understanding of supergravity theories is currently developed to the stage where all of the usual simplifying assumptions need be kept, so we have reexamined the ideas and constructed a class of attractive models (with a light higgsino) which are consistent with cosmological constraints and with a heavy-squark, light-gluino interpretation of the monojet data. In addition to finding some interesting models, we have also found that some new and exciting ways to detect supersymmetric signals and perhaps even a Higgs boson also arise.

Sect. 2 discusses how to obtain $S U(2) \times U(1)$ breaking in the more general theory we consider. Sect. 3 constrains parameters to obtain the spectrum with heavy squarks and light gluinos that we are interested in studying. Sect. 4 examines the general constraints on the relation between photino and gluino masses, and the extent to which they should be considered related. Sect. 5 examines a number of phenomenological implications of our model. Some are unexpected and possibly 
important and can be relevant in many models. Our lightest supersymmetric particle is a higgsino $\tilde{\mathrm{h}}$, so photinos produced at colliders decay, $\tilde{\gamma} \rightarrow \gamma \tilde{\mathrm{h}}$. Then monojet events will be accompanied by photons, and analyses will have to be reinterpreted. Also, the process $\mathrm{e}^{+} \mathrm{e}^{-}$(or $\mathrm{q} \overline{\mathrm{q}}$ ) $\rightarrow \mathrm{Z}^{0} \rightarrow \tilde{\mathrm{h}} \tilde{\mathrm{Z}}$, followed by $\tilde{\mathrm{Z}} \rightarrow \tilde{\mathrm{h}} \mathrm{H}^{0}$, occurs with a sizeable rate, possibly of order $1 \%$ of all $\mathrm{Z}^{0}$ decays, and leads to an excellent way of searching for a Higgs boson. Details of the general form of the low-energy theory are given in appendix A. Appendices B and C provide useful information relevant for the phenomenological analysis of our model.

\section{2. $S U(2) \times U(1)$ radiative breaking}

This section is devoted to the study of $\mathrm{SU}(2) \times \mathrm{U}(1)$ radiative breaking in the most general renormalizable low-energy effective theory obtained from the flat limit of $N=1$ supergravity with canonical kinetic terms, as described in appendix A. Notations, conventions and many results will be borrowed from ref. [8].

The supersymmetric superpotential $g$ which appears in (A.11) and (A.13), and is responsible for Yukawa couplings, is chosen as usual

$$
g=h_{1} U^{\mathrm{c}} Q \mathrm{H}_{2}+m_{4} \mathrm{H}_{1} \mathrm{H}_{2},
$$

where only the top Yukawa coupling has been kept in (2.1) since it is the only relevant one for the renormalization group equations [8] and $H_{1}, H_{2}$ denote the Higgs doublets giving mass to the matter fields. The supersymmetric mass $m_{4}$ which gives rise to a higgsino mass term, $m_{4} \tilde{H}_{1} \tilde{H}_{2}$, plays a crucial role for $\mathrm{SU}(2) \times \mathrm{U}(1)$ breaking in the usual low-energy theory $(w(\zeta) \equiv 1)$, if the top-quark mass is inside the experimental allowed range, $30 \mathrm{GeV} \leqslant m_{1} \leqslant 50 \mathrm{GeV}$. Furthermore, $m_{4}$ cannot be much smaller than the electroweak scale (since it governs the electroweak breaking); this means that in the usual case the higgsino cannot be made arbitrarily light so as to become the LSP. However, in the low-energy theory described in appendix $\mathrm{A}$ there is another (soft-breaking) superpotential $h$ which can be written, without loss of generality, as

$$
h=m_{4}^{\prime} H_{1} H_{2}
$$

(the inclusion of a trilinear coupling in (2.2), as in (2.1), would only change the definition of the $A$-parameter in (A.11), which is dependent on the super-Higgs mechanism and, therefore, arbitrary.) The new mass parameter $m_{4}^{\prime}$ is independent of $m_{4}$, and will be responsible for the electroweak breaking of the low-energy theory as we will see in what follows; it is not related to the higgsino mass which can be put small at will without paying any penalty. In short, the essence of the low-energy theory given by (A.11) is the separation of the electroweak breaking parameter from the higgsino mass and, as a bonus, we get one more parameter to describe the 
low-energy phenomenology. To put if differently, there is no known physical reason to couple the Higgsino mass and the electroweak breaking. It is an artificial restriction which we will not impose until an argument emerges in the future to suggest it.

The scalar potential (A.11) can be written, using (2.1) and (2.2), as

$$
V=\left|\frac{\partial g}{\partial y^{\alpha}}\right|^{2}+m_{3 / 2}^{2}\left|y^{a}\right|^{2}+\left(A h_{\mathrm{t}} m_{3 / 2} U^{\mathrm{c}} Q H_{2}+m_{3}^{2} H_{1} H_{2}+\text { h.c. }\right)+\frac{1}{2} D^{\alpha} D^{\alpha},
$$

where $y^{a}$ are all the scalar fields and the parameters $A$ and $m_{3}^{2}$ have, as boundary values,

$$
\begin{aligned}
A\left(M_{\mathrm{X}}\right) & =A \\
m_{3}^{2}\left(M_{\mathrm{X}}\right) & =\left(B m_{4}\left(M_{\mathrm{X}}\right)+C m_{4}^{\prime}\left(M_{\mathrm{X}}\right)\right) m_{3 / 2}
\end{aligned}
$$

where $A, B$ and $C$ are the parameters defined in (A.11).

Hereafter, and for the purpose of renormalization group equations, we will use as independent soft-breaking parameters $A$ and $m_{3}^{2}$.

The scalar potential involving the neutral components of Higgs scalars, which will be denoted by $H_{1}$ and $H_{2}$, is given by

$$
\begin{aligned}
V\left(H_{1}, H_{2}\right)= & m_{1}^{2}\left|H_{1}\right|^{2}+m_{2}^{2}\left|H_{2}\right|^{2}-m_{3}^{2}\left(H_{1} H_{2}+H_{1}^{\dagger} H_{2}^{\dagger}\right) \\
& +\frac{1}{8}\left(g_{2}^{2}+g_{1}^{2}\right)\left(\left|H_{1}\right|^{2}-\left|H_{2}\right|^{2}\right)^{2}+\Delta V_{\text {rad }},
\end{aligned}
$$

where $\Delta V_{\text {rad }}$ is the one-loop correction [8] to the effective potential and parameters $m_{1}^{2}$ and $m_{2}^{2}$ have as boundary conditions

$$
m_{1}^{2}\left(M_{\mathrm{X}}\right)=m_{2}^{2}\left(M_{\mathrm{X}}\right)=m_{3 / 2}^{2}+m_{4}^{2}\left(M_{\mathrm{X}}\right) .
$$

We define the vacuum expectation values $\left\langle H_{i}\right\rangle \equiv v_{i}$ and introduce the notation $v_{1}=v \cos \theta, v_{2}=v \sin \theta$. Neglecting the radiative corrections in (2.5), the tree-level potential is minimized for [3]

$$
v^{2}=\frac{2\left(m_{2}^{2}-m_{1}^{2}\right)-2\left(m_{1}^{2}+m_{2}^{2}\right) \cos 2 \theta}{\left(g_{2}^{2}+g_{1}^{2}\right) \cos 2 \theta} .
$$

As was shown in ref. [8] all renormalization group equations can be integrated analytically if the top-quark is sufficiently light, actually for $m_{\mathrm{t}}<\mathrm{O}(100) \mathrm{GeV}$. In that case it is convenient to choose as an input parameter, instead of $h_{\mathrm{t}}\left(M_{\mathrm{X}}\right)$ or 
$h_{\mathrm{t}}\left(M_{\mathrm{w}}\right)$

$$
r^{2}=\int_{0}^{\log \left(M_{\mathrm{x}}^{2} / M_{\mathrm{w}}^{2}\right)} \mathrm{d} t\left(\frac{h_{\mathrm{t}}}{4 \pi}\right)^{2}
$$

related to the top-quark mass by

$$
\left(\frac{m_{\mathrm{t}}}{M_{\mathrm{W}}}\right)^{2}=2 \sin ^{2} \theta\left(N \frac{r^{2}}{1+6 r^{2}}\right)
$$

where $N$ is the parameter defined in ref. [8] which takes the value $N=21.83$ in the minimal model ${ }^{\star}$. Actually $r^{2}$ is a small parameter: for $m_{\mathrm{t}}=\mathrm{O}(40) \mathrm{GeV}, r^{2}=$ $\mathrm{O}\left(10^{-2}\right)$.

The $\mathrm{SU}(2) \times \mathrm{U}(1)$ breaking condition, $M_{\mathrm{Z}}^{2}=\frac{1}{2}\left(g_{2}^{2}+g_{1}^{2}\right) v^{2}$, gives, using (2.7) and the analytic solution of the renormalization group equations [8],

$\cos 2 \theta=-\frac{3 r^{2}\left[3+\left(C_{\mathrm{Q}}+C_{\mathrm{U}}+C_{\mathrm{L}}\right) \xi^{2}+A^{2}\right]}{\left(1+6 r^{2}\right)\left[2\left(\hat{m}_{4}^{2}+1+C_{\mathrm{L}} \xi^{2}\right)+\hat{M}_{\mathrm{Z}}^{2}\right]-3 r^{2}\left[3+\left(C_{\mathrm{Q}}+C_{\mathrm{U}}+C_{\mathrm{L}}\right) \xi^{2}+A^{2}\right]}$

where all hatted masses are scaled with $m_{3 / 2} ; \xi=m_{\tilde{v}} / m_{3 / 2}, m_{\tilde{v}}$ being a common gaugino Majorana mass at $M_{\mathrm{X}}$ and the other parameters take the values, in the minimal model [8]: $C_{\mathrm{Q}}=5.290, C_{\mathrm{U}}=4.893, C_{\mathrm{L}}=0.496, C_{\mathrm{E}}=0.149, C_{\mathrm{D}}=4.843$.

Using the relation

$$
\sin 2 \theta=\frac{2 m_{3}^{2}}{m_{1}^{2}+m_{2}^{2}}
$$

and the breaking condition (2.10) we get the value of $m_{3}^{2}\left(M_{\mathrm{W}}\right)$ consistent with electroweak breaking

$$
\hat{m}_{3}^{2}=\sin 2 \theta\left\{\hat{m}_{4}^{2}+1+C_{\mathrm{L}} \xi^{2}-\frac{3 r^{2}}{1+6 r^{2}}\left[3+\left(C_{\mathrm{Q}}+C_{\mathrm{U}}+C_{\mathrm{L}}\right) \xi^{2}+A^{2}\right]\right\} .
$$

For a particular choice of the parameters $m_{3 / 2}, \xi, A, m_{4}$ and $r^{2}$ we get $\cos 2 \theta$ from (2.10) (and, therefore, $m_{1}$ from (2.9)) and $m_{3}^{2}$ from (2.12). Thus $m_{3}^{2}$ is * The minimal supersymmetric model, with three generations of quarks and leptons and two Higgs
doublets, with the low-energy input $\alpha_{3}^{-1}\left(M_{\mathrm{w}}\right)=9.9, \alpha^{-1}\left(M_{\mathrm{w}}\right)=127.54$, has the GUT predictions:

$$
\begin{gathered}
M_{\mathrm{X}}=5.23 \times 10^{15} \mathrm{GeV}, \quad \sin ^{2} \theta_{\mathrm{W}}\left(M_{\mathrm{W}}\right)=0.236 . \\
\alpha_{\mathrm{G} \text { UUT }}^{-1}=25.1, \quad \alpha_{1}^{-1}\left(M_{\mathrm{W}}\right)=58.5, \quad \alpha_{2}^{-1}\left(M_{\mathrm{W}}\right)=30.2 .
\end{gathered}
$$


determined by the breaking condition while $m_{4}$ and $A$ are just free parameters. This proves our statement that the electroweak breaking scale and the higgsino mass get decoupled.

For a small top-quark mass, $r^{2} \rightarrow 0$ and $\cos 2 \theta \rightarrow 0, \sin 2 \theta \rightarrow 1$, so that the breaking condition requires $m_{3}^{2}=\mathrm{O}\left(m_{3 / 2}^{2}\right)$ (while $m_{4}$ and $\xi$ can take any value, even zero). In that case we reach an almost flat direction which translates into a very light neutral Higgs. However, along this direction the radiative corrections are not negligible [8] and the true minimum has to be found by minimizing the total effective potential. A good approximation in many cases will be to neglect the tree-level contribution and minimize the one-loop corrections (pure dimensional transmutation mechanism); this will give a radiative mass larger than the tree-level mass. The positivity of this radiative mass is guaranteed by the stability condition [8]

$$
\hat{m}_{3}^{2}\left(M_{\mathrm{X}}\right)<1+\hat{m}_{4}^{2}\left(M_{\mathrm{X}}\right)
$$

which protects the diagonal direction $H_{1}=H_{2}$ in (2.5), not protected by $D$-terms.

Another condition, to prevent the appearance of an $\mathrm{SU}(3) \times \mathrm{U}(1)_{\mathrm{em}}$ breaking minimum at a scale $M_{\mathrm{W}} \leqslant \mu \leqslant M_{\mathrm{X}}$, has to be imposed. A necessary condition has been proved to be $[8,24]$

$$
A^{2}<3\left(\hat{m}_{\mathrm{Q}}^{2}+\hat{m}_{\mathrm{U}}^{2}+\hat{m}_{2}^{2}\right)
$$

which translates at $M_{\mathrm{X}}$ into

$$
A^{2}\left(M_{\mathrm{X}}\right)<3\left(3+\hat{m}_{4}^{2}\left(M_{\mathrm{X}}\right)\right)
$$

and at $M_{\mathrm{W}}$, using the breaking condition, into

$$
A^{2}<3 \frac{3+\left(C_{\mathrm{Q}}+C_{\mathrm{U}}+C_{\mathrm{L}}\right) \xi^{2}+\left(1+6 r^{2}\right) \hat{m}_{4}^{2}}{1+24 r^{2}} .
$$

In conclusion, for any set of parameters $m_{3 / 2}, m_{\tilde{v}}, m_{4}$ and $r^{2}$ satisfying conditions (2.14)-(2.16), $\cos 2 \theta$ (which depends on $m_{t}$ ) and $m_{3}^{2}$ are fixed by the radiative breaking condition (2.10) and (2.12) and a general analysis could be easily done. Instead, we will restrict ourselves to the case $\hat{m}_{4} \simeq 0, \xi \simeq 0$. This will be of interest for constructing a model where large $P_{\mathrm{T}}$ monojet events observed by UA1 could be explained by a mechanism with light $\tilde{\mathrm{g}}$ and heavy q (i.e. $m_{3 / 2} \simeq$ $\mathrm{O}(100) \mathrm{GeV}, m_{\tilde{\mathrm{g}}} \simeq \mathrm{O}(10) \mathrm{GeV}, m_{\tilde{\gamma}} \leqslant$ few $\mathrm{GeV}$ ), but without cosmological troubles (i.e. $m_{4} \leq \mathrm{O}(100) \mathrm{eV}$ ), as was explained in sect. 1. This approach will be discussed in some detail in the next section. 


\section{Application to heavy squark and light gluino scenarios}

We will apply now the general formalism of sect. 2 to the simplest scenario with heavy squarks, $\mathrm{O}(100) \mathrm{GeV}$, and light gluinos, $\mathrm{O}(5) \mathrm{GeV}$. In turn, this means photinos of $\mathrm{O}(1) \mathrm{GeV}$ in a minimal model; such light photinos have serious cosmological troubles if they are the LSP. In the usual models there is no way out of this situation, as was discussed in sect. 1 , since the higgsino mass is the electroweak breaking parameter. However, we have the freedom now to fix $m_{4} \leq \mathrm{O}(100) \mathrm{eV}$ so that photinos can decay before nucleosynthesis and no cosmological bound applies.

By choosing $m_{4}$ so small, we apparently have a naturainess problem due to the appearance of two very different mass scales: $m_{3}=m_{3 / 2} \approx 100 \mathrm{GeV}$ and $m_{4}$ $10^{-7} \mathrm{GeV}$. Suppose we initially fix $m_{4}=0$ and let the higgsino get a mass through loop corrections. The natural scale for $m_{4}$ is of order $g^{2} m_{\mathrm{W}}$, which is much larger than what we have assumed. For example, if the theory is embedded into a grand unified theory, then starting from $m_{4}=0$ we get an effective $m_{4} \neq 0$ from loop diagrams (refs. [4, 7, 26]) involving the doublet-triplet splitting mechanism (ref. [27]). It is possible in extended models (with more structure at the grand unified scale) to have a very light neutralino with mass of order $100 \mathrm{eV}$, as is found for the axino in ref. [25]. Unfortunately, the axino is very weakly coupled to all other matter and can be ignored when considering the phenomenology of supersymmetry at colliders. Whether it is possible in realistic models to obtain such a light higgsino, with couplings to matter of electroweak strength, naturally, is an open question. We will assume that $m_{4} \sim 10^{-7} \mathrm{GeV}$ is reasonable.

Neglecting $\hat{m}_{4}$ and $\xi^{2}$ in (2.10)-(2.12), the breaking condition now reads as

$$
\hat{m}_{3}^{2}=1-\frac{3}{2} r^{2}\left(A^{2}+3\right)=1+\left(1+\frac{1}{2} \hat{M}_{2}^{2}\right) \cos 2 \theta .
$$

By integration of the renormalization group equation for $m_{3}^{2},(1.2)$, we get

$$
\hat{m}_{3}^{2}=C_{4} \hat{m}_{3}^{2}\left(M_{\mathrm{X}}\right) \mathrm{e}^{-3 r^{2} / 2}
$$

where $C_{4}=1.365$. Using now the breaking condition (3.1) we get

$$
\hat{m}_{3}^{2}\left(M_{\mathrm{X}}\right)=\frac{1}{C_{4}}\left[1-\frac{3}{2} r^{2}\left(A^{2}+2\right)\right]<1,
$$

so that the stability condition (2.13) is automatically satisfied.

The color bound at $M_{\mathrm{X}}$ gives the usual constraint

$$
\left|A\left(M_{\mathrm{X}}\right)\right|<3,
$$


while the color bound at $M_{\mathrm{w}},(2.16)$, translates into

$$
A^{2}<\frac{9}{1+24 r^{2}}
$$

which, for $r^{2}=10^{-2}$, gives the bound $|A| \leqslant 2.7$.

Next we will write the supersymmetric spectrum, as in ref. [8], but including now the contributions from the $D$-terms which, although small, are larger than those coming from gaugino mass terms. We will neglect $m_{4}$ throughout.

(i) Sleptons (3 generations) and squarks (1st and 2 nd generation)

$$
\begin{aligned}
& m_{\hat{\ell}_{\mathrm{L}}}^{2}=m_{3 / 2}^{2}\left(1+C_{\mathrm{L}} \xi^{2}\right)-M_{\mathrm{Z}}^{2}\left(\frac{1}{2}-\sin ^{2} \theta_{\mathrm{W}}\right) \cos 2 \theta, \\
& m_{\hat{\ell}_{\mathrm{R}}}^{2}=m_{3 / 2}^{2}\left(1+C_{\mathrm{E}} \xi^{2}\right)-M_{\mathrm{Z}}^{2} \sin ^{2} \theta_{\mathrm{W}} \cos 2 \theta, \\
& m_{\hat{\nu}_{\mathrm{L}}}^{2}=m_{3 / 2}^{2}\left(1+C_{\mathrm{L}} \xi^{2}\right)+\frac{1}{2} M_{\mathrm{Z}}^{2} \cos 2 \theta
\end{aligned}
$$

and

$$
\begin{aligned}
& m_{\tilde{\mathrm{u}}_{\mathrm{L}}}^{2}=m_{3 / 2}^{2}\left(1+C_{\mathrm{Q}} \xi^{2}\right)-M_{\mathrm{Z}}^{2}\left(-\frac{1}{2}+\frac{2}{3} \sin ^{2} \theta_{\mathrm{W}}\right) \cos 2 \theta, \\
& m_{\mathrm{U}_{\mathrm{R}}}^{2}=m_{3 / 2}^{2}\left(1+C_{\mathrm{U}} \xi^{2}\right)+M_{\mathrm{Z}}^{2}\left(\frac{2}{3} \sin ^{2} \theta_{\mathrm{W}}\right) \cos 2 \theta, \\
& m_{\mathrm{d}_{\mathrm{L}}}^{2}=m_{3 / 2}^{2}\left(1+C_{\mathrm{Q}} \xi^{2}\right)-M_{\mathrm{Z}}^{2}\left(\frac{1}{2}-\frac{1}{3} \sin ^{2} \theta_{\mathrm{W}}\right) \cos 2 \theta, \\
& m_{\mathrm{d}_{\mathrm{R}}}^{2}=m_{3 / 2}^{2}\left(1+C_{\mathrm{D}} \xi^{2}\right)-M_{\mathrm{Z}}^{2}\left(\frac{1}{3} \sin ^{2} \theta_{\mathrm{W}}\right) \cos 2 \theta,
\end{aligned}
$$

where the parameter $\xi$ can be expressed in terms of the gluino mass $m_{\tilde{B}}$

$$
\xi^{2} m_{3 / 2}^{2}=0.16 m_{\tilde{\mathrm{g}}}^{2}
$$

by using eq. (4.1).

(ii) Squarks of the third generation

$$
\begin{aligned}
& m_{\mathrm{b}_{\mathrm{R}}}^{2}=m_{3 / 2}^{2}\left(1+C_{\mathrm{D}} \xi^{2}\right)-M_{\mathrm{Z}}^{2}\left(\frac{1}{3} \sin ^{2} \theta_{\mathrm{W}}\right) \cos 2 \theta, \\
& m_{\mathrm{b}_{\mathrm{L}}}^{2}=m_{3 / 2}^{2}\left(1+C_{\mathrm{Q}} \xi^{2}\right)-\varepsilon-M_{\mathrm{Z}}^{2}\left(\frac{1}{2}-\frac{1}{3} \sin ^{2} \theta_{\mathrm{W}}\right) \cos 2 \theta, \\
& m_{\mathrm{t}_{\mathrm{h}, \mathrm{l}}}^{2}=\frac{1}{2}\left[m_{\mathrm{Q}}^{2}+m_{\mathrm{U}}^{2}+2 m_{\mathrm{t}}^{2} \pm \sqrt{\left(m_{\mathrm{Q}}^{2}-m_{\mathrm{U}}^{2}\right)^{2}+4 A^{2} m_{\mathrm{t}}^{2} m_{3 / 2}^{2}}\right],
\end{aligned}
$$

where

$$
\begin{aligned}
& m_{\mathrm{Q}}^{2}=m_{3 / 2}^{2}\left(1+C_{\mathrm{Q}} \xi^{2}\right)-\varepsilon-M_{\mathrm{Z}}^{2}\left(-\frac{1}{2}+\frac{2}{3} \sin ^{2} \theta_{\mathrm{W}}\right) \cos 2 \theta, \\
& m_{\mathrm{U}}^{2}=m_{3 / 2}^{2}\left(1+C_{\mathrm{U}} \xi^{2}\right)-2 \varepsilon-M_{\mathrm{Z}}^{2}\left(\frac{2}{3} \sin ^{2} \theta_{\mathrm{W}}\right) \cos 2 \theta
\end{aligned}
$$


and

$$
\varepsilon=\frac{r^{2}}{1+6 r^{2}}\left[3+A^{2}+\left(C_{\mathrm{Q}}+C_{\mathrm{U}}+C_{\mathrm{L}}\right) \xi^{2}\right] m_{3 / 2}^{2}
$$

comes from the integration of the top Yukawa coupling.

(iii) Higgses. There are three neutral higgses with masses

$$
\begin{aligned}
m_{H_{1}}^{2} & =m_{1}^{2}+m_{2}^{2} \\
m_{H_{2,3}}^{2} & =\frac{1}{2}\left[m_{H_{1}}^{2}+M_{Z}^{2} \pm \sqrt{\left(m_{H_{1}}^{2}+M_{Z}^{2}\right)^{2}-4 m_{H_{1}}^{2} M_{Z}^{2} \cos ^{2} 2 \theta}\right] .
\end{aligned}
$$

In the limit $\cos 2 \theta \rightarrow 0$ we get

$$
m_{H_{2}}^{2}=m_{H_{1}}^{2}+M_{Z}^{2}
$$

and $m_{H_{3}}^{2}=0$, which means that the potential is flat along this direction. In that case radiative corrections have to be taken into account in (2.5) and the minimization of $\Delta V_{\text {rad }}$ gives a non-vanishing radiative mass [8]

$$
m_{\mathrm{rad}}^{2} \simeq\left\{\frac{3 \alpha_{2}}{4 \pi}\left[1+\left(A^{2}+2\right)\left(\frac{m_{\mathrm{t}}}{M_{\mathrm{w}}}\right)^{2}\right]+\frac{3 \alpha_{1}}{20 \pi}\right\} m_{3 / 2}^{2},
$$

where the contribution from gauginos has been neglected. In fact, the ratio $\Lambda=$ $m_{\mathrm{rad}}^{2} / m_{H_{3}}^{2}$ is a naive measure of the validity of the dimensional transmutation approximation [8] or the tree-level approximation [7,20]. In all cases studied in this paper we have found that $\Lambda \gg 1$ which means that the dimensional transmutation mechanism is a good approximation and that the corresponding neutral Higgs is heavier than what we would have obtained from the tree-level approximation [20].

There is also a charged Higgs with mass

$$
m_{\mathrm{H}^{ \pm}}^{2}=m_{H_{1}}^{2}+M_{\mathrm{W}}^{2}
$$

(iv) Charginos. The charged winos combine with charged higgsinos and form a couple of Dirac particles with masses

$$
m_{\mathrm{H} \pm}^{2}=M_{\mathrm{W}}^{2} \pm \sqrt{M_{\mathrm{W}}^{2}\left(\left(\frac{\alpha_{2}}{\alpha_{3}}\right)^{2} m_{\tilde{\mathrm{g}}}^{2}+M_{\mathrm{W}}^{2} \cos ^{2} 2 \theta\right)},
$$

where we have used the approximation $\left(\alpha_{2} / \alpha_{3}\right) m_{\hat{\mathrm{g}}} \ll M_{\mathrm{w}}$.

(v) Neutralinos. There are four neutral fermions, $\tilde{W}^{3}, \tilde{B}, \tilde{H}_{1}^{0}$ and $\tilde{H}_{2}^{0}$ which mix in a four-by-four mass matrix [28]. In the limit $\cos 2 \theta \rightarrow 0$ there is a pure higgsino 
state [see (B.3)]

$$
\tilde{h}=\sqrt{\frac{1}{2}}\left(\tilde{H}_{1}^{0}+\tilde{H}_{2}^{0}\right)
$$

with a mass equal to $m_{4}$. Furthermore, if $\left(\alpha_{1} / \alpha_{3}\right) m_{\tilde{\mathrm{g}}},\left(\alpha_{2} / \alpha_{3}\right) m_{\tilde{\mathrm{g}}} \ll M_{\mathrm{W}}$ (which is the case in the class of models we are considering here) there is an approximately pure photino state $\tilde{\gamma}=\tilde{W}^{3} \sin \theta_{\mathrm{W}}+\tilde{B} \cos \theta_{\mathrm{W}}$ with a mass

$$
m_{\tilde{\gamma}}=\frac{8}{3} \sin ^{2} \theta_{\mathrm{W}} \frac{\alpha_{2}}{\alpha_{3}} m_{\tilde{\mathrm{g}}}
$$

and a Dirac spinor $\tilde{Z}$ with a mass $M_{Z}$. Actually the Dirac spinor $\tilde{Z}$ is a combination of two Majorana spinors $\tilde{Z}_{ \pm}$with masses

$$
m_{\tilde{\mathrm{Z}}_{ \pm}}=M_{\mathrm{Z}} \pm \Delta
$$

where in the minimal model considered here,

$$
\Delta=\left|0.038 m_{\tilde{\mathrm{g}}}-0.893 m_{\tilde{\gamma}}\right|
$$

How many free parameters does the supersymmetric spectrum depend on? In principle, there are five free parameters: $m_{3 / 2}, m_{\tilde{B}}, A, m_{4}$ and $m_{3}^{2}$. If we want to adhere to a particular supersymmetric interpretation of UA1 monojets, i.e. a particular value of $m_{\tilde{\mathrm{q}}}$ and $m_{\tilde{\mathrm{g}}}$, we fix $m_{3 / 2}$ and $m_{\tilde{\mathrm{g}}}$. The condition for $\mathrm{SU}(2) \times \mathrm{U}(1)$ breaking determines $m_{3}^{2}$. We are left with two free parameters, $A$ and $m_{4}$, which have to respect the color bound (2.15), (2.16). In the particular scenario of heavy squarks and light gluinos, the cosmological constraint on relic higgsinos suggests fixing $m_{4} \leq \mathrm{O}(100) \mathrm{eV}$ in order to avoid the cosmological bounds [29]. Thus the whole spectrum depends only on one free parameter $A$ which has to satisfy the bound $|A| \leqslant 2.7$ to prevent the appearance of color and/or electric charge breaking minima. In table 1 we give the numerical predictions for the supersymmetric spectrum for $m_{3 / 2}=110 \mathrm{GeV}$ and $m_{\tilde{\mathrm{g}}}=5 \mathrm{GeV}$, and several values of $A$. Two comments are interesting: (a) The only squarks which are split in mass are the $\mathrm{t}$-squarks due to the non-diagonal $\left(\mathrm{t}_{\mathrm{L}}^{*} \tilde{\mathfrak{t}}_{\mathrm{R}}\right)$ contributions, proportional to $A m_{\mathrm{t}} m_{3 / 2}$. However, the UA1 report of the top quark at $m_{\mathrm{t}} \simeq 40 \mathrm{GeV}$ from $\mathrm{t} \rightarrow \mathrm{b} \ell \nu$ indicates that the mode $\mathrm{t} \rightarrow \tilde{\mathrm{t}} \tilde{\gamma}$ is suppressed kinematically (otherwise it would be the dominant decay mode), i.e. $m_{\mathfrak{t}_{e}}>m_{\mathrm{t}}$. This imposes a further constraint on the value of the $A$ parameter which is found to be bounded by $|A| \leq 2.4$. (b) The dimensional transmutation mechanism is a good approximation for the radiative breaking. In fact, the parameter $\Lambda$ introduced below eq. (3.15) takes the values, as $A$ varies from 0 to 2.4 in table $1, \Lambda(0)=21.3, \Lambda(1)=13.8, \Lambda(2)=6.5$ and $\Lambda(2.4)=4.5$. This makes the radiative masses in the table larger than those obtained [20] from the 
TABLE 1

Supersymmetric spectrum corresponding to the case: $m_{3 / 2}=110 \mathrm{GeV}$, $m_{\tilde{\mathrm{g}}}=5 \mathrm{GeV}, m_{\mathrm{t}}\left(m_{\mathrm{W}}\right)=39 \mathrm{GeV}, m_{\tilde{\mathrm{h}}}<100 \mathrm{eV}$

\begin{tabular}{|c|c|c|c|c|c|}
\hline $\begin{array}{l}A(\lambda \\
v_{1} / l \\
\cos 2 \\
\theta\end{array}$ & & $\begin{array}{c}0 \\
1.03 \\
-0.0335 \\
45.96^{\circ}\end{array}$ & $\begin{array}{c}1 \\
1.05 \\
-0.0447 \\
46.28^{\circ}\end{array}$ & $\begin{array}{c}2 \\
1.08 \\
-0.0782 \\
47.24^{\circ}\end{array}$ & $\begin{array}{c}2.4 \\
1.10 \\
-0.0979 \\
47.81^{\circ}\end{array}$ \\
\hline \multirow[t]{3}{*}{3 generations } & $\tilde{\ell}_{\mathrm{L}}$ & 110.3 & 110.4 & 110.8 & 111.0 \\
\hline & $\tilde{\ell}_{\mathrm{R}}$ & 110.3 & 110.4 & 110.7 & 110.9 \\
\hline & $\tilde{\nu}_{\mathrm{L}}$ & 109.4 & 109.2 & 108.5 & 108.1 \\
\hline \multirow{4}{*}{2 generations } & $\tilde{u}_{\mathrm{L}}$ & 109.6 & 109.4 & 109.0 & 108.7 \\
\hline & $\tilde{\mathrm{u}}_{\mathrm{R}}$ & 109.8 & 109.7 & 109.5 & 109.4 \\
\hline & $\tilde{\mathrm{d}}_{\mathrm{L}}$ & 110.5 & 110.7 & 111.2 & 111.5 \\
\hline & $\overline{\mathrm{d}}_{\mathrm{R}}$ & 110.1 & 110.1 & 110.2 & 110.3 \\
\hline \multirow[t]{4}{*}{ 3rd generation } & $\tilde{b}_{\mathrm{L}}$ & 109.2 & 108.8 & 108.1 & 107.0 \\
\hline & $\tilde{b}_{R}$ & 110.1 & 110.1 & 110.2 & 110.3 \\
\hline & $i_{h}$ & 114.5 & 130.2 & 143.4 & 147.9 \\
\hline & $\tilde{\mathrm{t}}_{1}$ & 113.2 & 92.7 & 61.8 & 42.4 \\
\hline \multirow[t]{3}{*}{ neutral higgses } & $\mathrm{H}_{1}$ & 152.1 & 150.9 & 147.4 & 145.3 \\
\hline & $\mathrm{H}_{2}$ & 177.2 & 176.2 & 173.2 & 171.5 \\
\hline & $\mathrm{H}_{\mathrm{rad}}^{2}$ & 12 & 13 & 15.5 & 16.2 \\
\hline charged higgs & $\mathrm{H}^{ \pm}$ & 173.2 & 172.2 & 169.1 & 167.4 \\
\hline \multirow{3}{*}{ charginos } & $\overline{\mathrm{H}}^{+}$ & 84.6 & 85.0 & 86.3 & 87.0 \\
\hline & $\tilde{\mathrm{H}}^{-}$ & 81.4 & 81.0 & 79.6 & 78.8 \\
\hline & $\tilde{\gamma}$ & 1.0 & 1.0 & 1.0 & 1.0 \\
\hline neutralinos & $\tilde{\mathrm{Z}}$ (Dirac) & 91 & 91 & 91 & 91 \\
\hline
\end{tabular}

All masses are in $\mathrm{GeV}$.

tree-level potential, (3.13). As a consequence this Higgs cannot be present in the decay products of the $T(\mathrm{~b} \bar{b})$ but only in toponium states.

\section{On the relationship between photino and gluino majorana masses}

In sect. 2 we have assumed a common gaugino Majorana mass at $M_{\mathrm{X}}, M_{1}=M_{2}$ $=M_{3} \equiv m_{\bar{v}}$, which scale, following the renormalization group equations, as

$$
M_{i}(Q)=\frac{\alpha_{i}(Q)}{\alpha_{G U T}} M_{i} .
$$

However, in $N=1$ supergravity gaugino Majorana masses appear only from the term

$$
\frac{1}{4} \mathrm{e}^{G / 2} G^{A}\left(G^{-1}\right){ }_{A}^{B} f_{\alpha \beta ; B} \lambda^{\alpha} \lambda^{\beta}+\text { h.c, }
$$


so that if $f_{\alpha \beta}=\delta_{a \beta}$ all gaugino masses vanish. In this way, since we did not want massless gauginos, we have so far implicitly assumed the usual simple ansatz

$$
f_{\alpha \beta}=A(z) \delta_{\alpha \beta}
$$

The gauge boson and gaugino kinetic energy terms in the lagrangian define $f_{\alpha \beta}$;

$$
L \sim \operatorname{Re} f_{\alpha \beta}\left(F_{\mu \nu}^{\alpha} F^{\beta \mu \nu}+\bar{\lambda}^{\alpha} \not \partial \lambda^{\beta}\right)+\cdots
$$

Eq. (4.3) leads to the equality of all gaugino masses ( $\tilde{\mathrm{g}}, \tilde{\mathrm{W}}, \tilde{\mathrm{B}}, \tilde{\mathrm{X}}$ and $\tilde{\mathrm{Y}}$ ) at $M_{\mathrm{X}}$ because it is $S U(5)$-invariant. However there is no reason (but simplicity) for the choice (4.3); in general $f_{\alpha \beta}$ has to transform as the symmetric product of two adjoint representations and $\left\langle f_{\alpha \beta}\right\rangle$ has to be $\mathrm{SU}(3) \times \mathrm{SU}(2) \times \mathrm{U}(1)$-invariant: these conditions are consistent with the ansatz [30]

$$
f_{\alpha \beta}=A(z) \delta_{\alpha \beta}+B(z) d_{\alpha \beta \gamma} \Sigma^{\gamma}
$$

where $\Sigma^{\gamma}$ is in the 24 representation of $\mathrm{SU}(5)$.

One should observe here that (4.4) is not the most general ansatz, but the philosophy of this paper is not to have the most general theory in the hidden sector but only to get the most general low-energy theory, so for our purposes eq. (4.4) is enough.

Now, since supersymmetry is spontaneously broken in the hidden sector (see appendix A), $G_{z}=3$, and the contribution of $G^{a a}$ to (4.2) can be neglected. Actually, $G^{a}=\mathrm{O}\left(M_{\mathrm{X}} / M\right) G^{2} \mathrm{O}\left(10^{-3}\right) G^{z}$. In this way the gaugino Majorana mass from (4.2) can be written as

$$
m_{\alpha \beta}=\frac{3}{4} m_{3 / 2}\left\langle f_{\alpha \gamma ; z} f_{\gamma \beta}^{-1}\right\rangle .
$$

If we do not want to change the GUT predictions $\left(M_{\mathrm{X}}, \sin ^{2} \theta_{\mathrm{W}}, m_{\mathrm{b}} / m_{\tau}\right)$ coming from $\alpha_{1}\left(M_{\mathrm{X}}\right)=\alpha_{2}\left(M_{\mathrm{X}}\right)=\alpha_{3}\left(M_{\mathrm{X}}\right)$ we have to impose the constraint

$$
\left\langle f_{\alpha \beta}\right\rangle \propto \delta_{\alpha \beta}, \quad \alpha, \beta=1, \ldots, 21, \ldots, 24
$$

(In more general cases, as those studied in ref. [31], condition (4.6) is not imposed.) This condition is satisfied if

$$
B\left(z_{0}\right)=0
$$

(It is not known how stable such a condition is, or whether it is natural. It would be even better not to impose such a condition, but for now we keep it.) 
Now when $\mathrm{SU}(5)$ is spontaneously broken to $\mathrm{SU}(3) \times \mathrm{SU}(2) \times \mathrm{U}(1),\left\langle\Sigma^{\gamma}\right\rangle=$ $\delta^{\gamma, 24} \Sigma_{0}\left(\Sigma_{0}=\mathrm{O}\left(M_{\mathrm{X}}\right)\right)$ and

$$
\left\langle f_{\alpha \beta, z}\right\rangle=a \delta_{\alpha \beta}+\sqrt{15} b d_{\alpha \beta 24},
$$

where $a=A^{\prime}\left(z_{0}\right)$ and $b=\sqrt{\frac{1}{15}} B^{\prime}\left(z_{0}\right) \Sigma_{0}$.

In this way, while $A(z)$ in (4.4) is any function such that $A^{\prime}\left(z_{0}\right)$ has the desired order of magnitude, $B(z)$ has to be chosen more carefully. Actually it has to satisfy conditions (4.7) and $B^{\prime}\left(z_{0}\right) \Sigma_{0}=\mathrm{O}(a)$. A possible choice could be $B(z)=(z-$ $\left.z_{0}\right) \exp (n z) A^{\prime}(z)$ with $n=\mathrm{O}(\mathrm{a}$ few $) \simeq 7 / \zeta_{0}$.

The substitution of (4.8) into (4.5) leads to the following gaugino masses at $M_{\mathrm{X}}$

$$
\begin{aligned}
& M_{3}=(a+2 b) m, \\
& M_{2}=(a-3 b) m, \\
& M_{1}=(a-b) m,
\end{aligned}
$$

where

$$
m=\frac{\sqrt{3}}{4 A\left(z_{0}\right)} m_{3 / 2}
$$

In the limit $\cos 2 \theta \rightarrow 0$ and $M_{1}, M_{2} \ll M_{\mathrm{W}}$, as in (3.19), the neutralino mass matrix gives the photino mass

$$
m_{\tilde{\gamma}}=\frac{\alpha_{2}}{\alpha_{\mathrm{GUT}}} \sin ^{2} \theta_{\mathrm{W}} \frac{1}{3}(8 a-14 b) m,
$$

while the gluino mass is

$$
m_{\overline{\mathrm{g}}}=\frac{\alpha_{3}}{\alpha_{\mathrm{GUT}}}(a+2 b) m
$$

To obtain (4.11, and 4.12) use has been made of (4.9).

Since gaugino masses depend on two free parameters $(a, b)$, depending on the hidden sector, we can use them to fix a particular relation between $m_{\tilde{\gamma}}$ and $m_{\tilde{\mathrm{g}}}$. We introduce a parameter $\lambda$ such that

$$
m_{\tilde{\gamma}}=\lambda \frac{8}{3} \sin ^{2} \theta_{\mathrm{w}}\left(\frac{\alpha_{2}}{\alpha_{3}} m_{\tilde{\mathrm{g}}}\right)
$$

where $\lambda=1$ corresponds to eq. (3.19). Using (4.13) in (4.11), (4.12) leads to

$$
a=\frac{14+16 \lambda}{8(1-\lambda)} b
$$


Once we fix $\lambda$ (see sect. 5), all gaugino masses are arbitrary, since they depend on the $b$-parameter, but scale as

$$
\frac{M_{3}}{15}=\frac{M_{2}}{20 \lambda-5}=\frac{M_{1}}{12 \lambda+3}
$$

so that all of them are fixed once we fix the gluino mass. As particular cases of (4.15) we find:

(i) For $\lambda=1$, i.e. $b=0$ (see eq. (4.14)),

$$
M_{1}=M_{2}=M_{3},
$$

which corresponds to the ansatz (4.3).

(ii) For $\lambda=-\frac{1}{2}, a=\frac{1}{2} b$

$$
m_{\bar{\gamma}}=\frac{4}{3} \sin ^{2} \theta_{W}\left(\frac{\alpha_{2}}{\alpha_{3}} m_{\tilde{\mathrm{g}}}\right)
$$

as observed in ref. [31], and

$$
M_{3}: M_{2}: M_{1}=5:-5:-1
$$

This case corresponds to zero lepto-quark gaugino Majorana masses [30]

$$
M_{\tilde{\mathbf{x}}, \tilde{y}}=\left(a-\frac{1}{2} b\right) m=0
$$

as required in some particular models.

(iii) For $\lambda \ll 1$, the photino mass is very small, and the limiting scaling behavior of $M_{i}$ is

$$
M_{3}: M_{2}: M_{1}=15:-5: 3
$$

(iv) Since $\lambda=(1-7 b / 4 a) /(1+2 b / a)$, there are significant changes in $m_{\tilde{\gamma}} / m_{\overline{\mathrm{g}}}$ even for $b / a \ll 1$. For example, $b / a=-0.25$ gives $\lambda=2.875$ and $b / a=+0.25$ gives $\lambda=0.375$.

In general the radiative breaking of $\mathrm{SU}(2) \times \mathrm{U}(1)$ should be reanalyzed in each particular case; in particular the coefficients $C_{i}(i=\mathrm{Q}, \mathrm{U}, \mathrm{D}, \mathrm{L}, \mathrm{E}, 4)$ in sect. 3 will change. However the numerical application to the light gluinos scenario remains unchanged since the renormalization due to gaugino masses was negligible.

\section{Phenomenological implications}

The theoretical and experimental constraints imposed so far have left the parameter $A$ essentially free, with $0 \leqslant|A| \leqslant 2.4$, and the photino and higgsino masses 

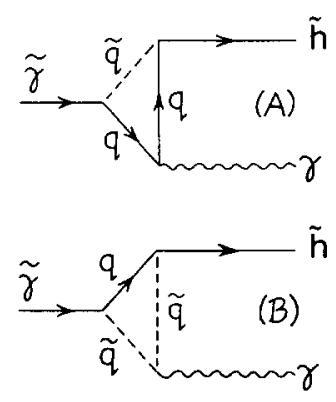

(a)
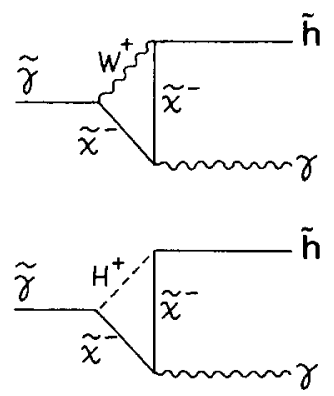
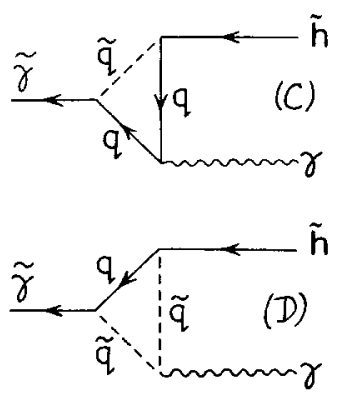

(D)
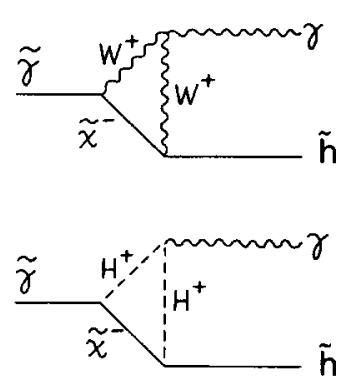

(b)

Fig. 1. Diagrams which contribute (in the unitary gauge) to the decay $\tilde{\gamma} \rightarrow \gamma \tilde{\mathrm{h}}$. In (a) one must sum over all possible flavours in the loop. In (b) $\mathrm{H}^{+}$is the charged Higgs, (3.16), and $\tilde{\chi}^{-}$the chargino eigenstate,

(3.17).

undetermined. There are two solutions consistent with the cosmological constraints [29]. Either $m_{\tilde{\mathrm{h}}} \leqslant 100 \mathrm{eV}$, or $m_{\overline{\mathrm{h}}} \geq m_{\mathrm{b}}$ so higgsino annihilates efficiently into $\mathrm{b} \overline{\mathrm{b}}$. In either case, we may arrange our parameters such that $m_{\tilde{\mathrm{h}}}<m_{\tilde{\gamma}}<m_{\tilde{\mathrm{g}}}$, since according to sect. $4 \mathrm{~m}_{\tilde{\gamma}} / \mathrm{m}_{\tilde{\mathrm{g}}}$ is a free parameter. For simplicity we take the higgsino to be massless in the discussion below. Let us consider a number of phenomenological implications.

(i) Since $h$ is lighter than $\tilde{\gamma}$, the decay $\tilde{\gamma} \rightarrow \gamma \tilde{\mathrm{h}}$ occurs at one loop, as in fig. 1 and is the dominant decay ${ }^{\star}$ of $\tilde{\gamma}$. (The decay $\tilde{\gamma} \rightarrow \tilde{h} q \bar{q}$ is suppressed by the weak $\tilde{h} q \tilde{q}$ coupling which is proportional to $m_{\mathrm{q}}$ ) In addition, diagrams such as fig. $1 \mathrm{~b}$ must be considered, but their contribution is suppressed in the model considered here, for reasons explained in appendix B. (This decay has also been computed in ref. [33].) We note that data from $\mathrm{e}^{+} \mathrm{e}^{-}$colliders [34] does not exclude photino decay via $\mathrm{e}^{+} \mathrm{e}^{-} \rightarrow \tilde{\gamma} \tilde{\gamma} \rightarrow \gamma \gamma+$ missing energy as present $\mathrm{e}^{+} \mathrm{e}^{-}$experiments are sensitive only for $m_{\tilde{c}} \leqslant 100 \mathrm{GeV}$ while our spectrum has ẽ with mass about $120 \mathrm{GeV}$. We obtain

\footnotetext{
* For earlier consideration of $\tilde{\gamma} \rightarrow \gamma+$ neutral light fermion, see ref. [32].
} 
for the photino width, in the limit of $m_{\mathfrak{t}}, m_{\tilde{t}} \gg m_{\tilde{\gamma}}$ :

$$
\Gamma=\frac{9 G_{\mathrm{F}} e_{\mathrm{t}}^{4} \alpha^{2} \cot ^{2} \theta}{16 \sqrt{2} \pi^{3}}\left[I_{1}+I_{2}-\frac{m_{\tilde{\gamma}}}{m_{\mathrm{t}}} \sin 2 \theta_{\mathrm{t}}\left(J_{1}-J_{2}\right)\right]^{2} M_{\tilde{\gamma}}^{3},
$$

where $e_{1}$ is the electric charge of the top quark, $\cot \theta=v_{1} / v_{2}, I_{i}, J_{i}$ are defined by

$$
\begin{aligned}
& I_{i}=\frac{1}{1-r_{i}}\left[1+\frac{r_{i}}{1-r_{i}} \log r_{i}\right], \\
& J_{i}=-\frac{1}{2}\left\{\frac{1+r_{i}}{2\left(1-r_{i}\right)^{2}}+\frac{r_{i}}{\left(1+r_{i}\right)^{3}} \log r_{i}\right\},
\end{aligned}
$$

with $r_{i}=\left(m_{\mathrm{t}_{i}} / \mathrm{m}_{\mathrm{t}}\right)^{2}$ and $\tilde{\mathrm{t}}_{1}, \tilde{\mathrm{t}}_{2}$ are the mass eigenstates defined in eq. (3.9c), $\theta_{\mathrm{t}}$ being the rotation from $\left(\tilde{t}_{L}, \tilde{t}_{R}\right)$ to $\left(\tilde{t}_{1}, \tilde{t}_{2}\right)$. In our model $\theta_{t} \simeq 45^{\circ}$.

In the particular case where there is no $\mathfrak{t}_{\mathrm{L}}, \mathfrak{t}_{\mathrm{R}}$ mixing, i.e. when $A=0, J_{1} \simeq J_{2}$ since $m_{\tilde{i}_{\mathrm{L}}} \approx m_{i_{\mathrm{R}}}, I_{1} \approx I_{2}=I$ and eq. (5.1) simplifies to

$$
\Gamma=\frac{9 G_{\mathrm{F}} e_{1}^{4} \alpha^{2} \cot ^{2} \theta}{4 \sqrt{2} \pi^{3}}|I|^{2} M_{\tilde{\gamma}}^{3}
$$

For the cases considered in table 1 a photino of energy $40 \mathrm{GeV}$ would travel

$$
\gamma c t=d\left[m_{\hat{\gamma}}(\mathrm{GeV})\right]^{4} \text { meters }
$$

where the parameter $d$ is a function of $A$. In particular $d=0.04,0.03,0.02$ and 0.01 meters for $A=0,1,2$ and 2.4 , respectively. For $m_{\tilde{\gamma}} \leqslant 0.25 \mathrm{GeV}$ all photinos would escape a collider detector while for $m_{\tilde{\gamma}} \geq 0.5 \mathrm{GeV}$ all photinos would decay within the detector. Since the decay $\tilde{\gamma} \rightarrow \gamma \tilde{h}$ gives a flat energy spectrum from 0 to $E_{\bar{\gamma}}$ for the $\tilde{h}$, some expected monojet events would have an energetic $\tilde{h}$ and remain monojet events, while others would have $E(\tilde{h}) \ll E(\tilde{\gamma})$ and not pass the UAl missing energy cuts. Events with isolated hard photons would occur, some with large accompanying missing momentum. If the $\tilde{\mathrm{g}}$ is light as in the model presented here, then the analysis of ref. [13] implies that the photino decays must be prompt. In other circumstances one could imagine that an interesting possibility could arise if missing energy events due to supersymmetry are eventually found. Depending on the photino lifetime, it could happen that a large number of $\tilde{\gamma}$ 's would escape the detector before decaying, giving rise to isolated photons which suddenly appear some meters from the collider detector. They could be searched for by surrounding part of the large angle region of the detector at some distance with a photon detector. The photons would point back to the interaction approximately, and occur in timing coincidence with the 
missing energy event. If such a situation occurred, essentially every photino could be detected, instead of having to wait decade(s) as with neutrinos to find explicitly the carrier of missing momentum.

If we take the model seriously, then observation of the photons would not only confirm the entire viewpoint considered here, it would also allow determination of $m_{\tilde{\gamma}}-m_{\tilde{\mathrm{h}}}$, and probably also fix $m_{\tilde{\mathrm{g}}}$ and $m_{\tilde{\gamma}}$.

It should also be noted that if $m_{\tilde{\gamma}} \geqslant m_{\tilde{\mathrm{g}}}$ there is little effect on the analysis of collider experiments since $\tilde{\mathrm{g}} \rightarrow \mathrm{q} \overline{\mathrm{q}} \tilde{\mathrm{h}}$. The $\tilde{\mathrm{g}}$ lifetime increases, and beam dump analyses no longer apply, so that a light $\tilde{\mathrm{g}}$ (below $4-5 \mathrm{GeV}$ ) is not excluded, and heavy decaying $\tilde{\mathrm{g}}$ would behave as before.

(ii) In our model, and in fact in a large class of supersymmetric theories, a new opportunity to discover a Higgs boson presents itself. Rather generally, there will be a neutralino mass eigenstate, which we can call $\tilde{Z}_{-}$, that has mass $m_{\tilde{Z}_{-}} \leqslant m_{Z}$ (see eq. (3.20)). Then the decay $Z \rightarrow \tilde{h} \tilde{Z}_{-}$occurs at tree level with a strength of order the usual $Z \rightarrow \overline{f f}$ modes (such as $Z \rightarrow \mu^{+} \mu^{-}$) suppressed only by phase space. Once that decay has occurred, the $\tilde{Z}_{-}$can decay via $\tilde{Z}_{-} \rightarrow \tilde{\mathrm{h}} \mathrm{H}^{0}$, or $\tilde{\mathrm{Z}}_{-} \rightarrow \mathrm{q} \overline{\mathrm{q}} \tilde{\mathrm{g}}$ or $\tilde{\mathrm{Z}}_{-} \rightarrow \tilde{\mathrm{h} f} \bar{f}$. These may be comparable in size, and the $\tilde{\mathrm{h}} \mathrm{H}^{0}$ mode may dominate, essentially independently of $m_{\mathrm{H}^{\circ}}$ if $m_{\mathrm{H}^{0}} \leq 60-70 \mathrm{GeV}$. Thus it can happen that

$$
\mathrm{e}^{+} \mathrm{e}^{-} \rightarrow \mathrm{Z}^{0} \rightarrow \tilde{\mathrm{h}} \tilde{\mathrm{h}} \mathrm{H}^{0}
$$

occurs at the level of $10^{-3}$ of all $Z^{0}$ decays. This gives a very clear signature. In our model $12 \mathrm{GeV} \leqslant m_{\mathrm{H}} \leqslant 15 \mathrm{GeV}$, so $\mathrm{H} \rightarrow \mathrm{b} \vec{b}$. This could be another way to detect a Higgs boson [35]. Some of the relevant rates are given in appendix $C$.

(iii) Several interesting $Z^{0}$ decays occur or are absent: (a) $Z \rightarrow \tilde{g} \tilde{g}$ has a branching ratio [36] of order $10^{-5}$ which varies significantly as $A$ varies from 0 to 2.4, so this mode can be detected eventually and can determine $A$ [36]. (b) The effect on $\Gamma_{\mathrm{Z}}$ is small, as no squark or slepton modes are energetically allowed, $Z \rightarrow \tilde{h} \tilde{h}$ is suppressed by a factor $\left(v_{2}-v_{1}\right)^{2} / v^{2}$, and so the largest new mode is $\tilde{\mathrm{h}} \tilde{\mathrm{Z}}$.

(iv) A $\mathrm{t}$ pair can be directly produced and detected at a hadron collider. The cross section is about one quarter that for a quark of the same mass, but the signature could be better because of the $\tilde{\gamma}$ or $\tilde{h}$ in the decay. Measuring $m_{\overline{\mathrm{t}}}$, from the event structure or the production cross section, determines $A$.

(v) There should be no direct production of any sleptons or squarks at SLC, LEP, HERA or TRISTAN in our model.

(vi) The gluino has a branching ratio

$$
\tilde{\mathrm{g}} \rightarrow \mathrm{g} \tilde{\gamma},
$$

which can be as large as 3\% in our model [37]. The branching ratio determines $m_{i}$ and $A$ if it is measured, which could be possible when several hundred monojet events are available. 
(vii) In the photon counting experiments

$$
\mathrm{e}^{+} \mathrm{e}^{-} \rightarrow \gamma+\text { missing energy, }
$$

there will be no signal from the usual diagrams since $m_{\tilde{\mathrm{e}}} \simeq 120 \mathrm{GeV}$, but some small contribution can occur from the higgsino final state if $v_{1} \neq v_{2}$.

(viii) As far as we are aware, no interesting flavor changing neutral current effects are induced.

(ix) If $\tilde{h}$ is the lightest supersymmetric particle and $m_{\tilde{h}} \leq 100 \mathrm{eV}, \tilde{\mathrm{h}}$ is a good candidate for hot dark matter, and for contributing to stellar energy loss. Since the higgsinos are more weakly coupled to matter than ordinary neutrinos, they fall out of thermal equilibrium earlier. Actually their decoupling temperature is found to be: $T_{\mathrm{D}}=17.3 \mathrm{MeV}, 14.3 \mathrm{MeV}, 9.8 \mathrm{MeV}$ and $8.5 \mathrm{MeV}$ for the different cases considered in table 1, $A=0,1,2$ and 2.4. Nevertheless higgsinos are still too strongly coupled to matter to become candidates to warm dark matter. (If $m_{\tilde{\mathrm{h}}} \simeq m_{\mathrm{b}}$, then $\tilde{\mathrm{h}}$ may be a good candidate for cold dark matter.)

(x) In a paper by Enqvist, Masiero and Nanopoulos [38], proton decay through dimension-five operators is confronted with some popular supergravity explanations of UA1 monojets. In particular in the minimal model, $M_{\mathrm{X}} \simeq 10^{16} \mathrm{GeV}$, if $M_{\tilde{\mathrm{q}}} \simeq$ $10^{2} \mathrm{GeV}$ and $M_{\tilde{\mathrm{g}}} \simeq 5 \mathrm{GeV}$, they find that present experimental bounds on proton decay translate into the bound $m_{4} \leq 3.7 \mathrm{GeV}$. While this bound is clearly incompatible with $\mathrm{SU}(2) \times \mathrm{U}(1)$ in usual models, as explained in sect. 1 of this paper, it is indeed compatible (within the errors inherent to this kind of bounds) with our general mechanism and, in particular, with the two proposed models: $m_{4} \simeq$ $\mathrm{O}(100) \mathrm{eV}$ or $m_{4} \simeq m_{\mathrm{b}}$.

\section{Conclusion}

We propose a general class of low-energy supergravity models where some of the usual simplifying assumptions in the hidden sector are omitted. Since soft-breaking parameters are considered as boundary conditions in the low-energy theory (independently of the particular super-Higgs mechanism where they come from) we are led to consider the hidden sector leading to a general low-energy effective theory. The result is a theory where the electroweak breaking is decoupled from the supersymmetric higgsino mass parameters and the photino-to-gluino mass ratio is not rigidly fixed. This theory has two more free parameters than the minimal one: the masses of the higgsino and the photino. This additional freedom may be welcome for phenomenological purposes in many models, and in any case it cannot be avoided without arbitrary assumptions.

We have studied $\mathrm{SU}(2) \times \mathrm{U}(1)$ breaking and found it is easier to implement than in minimal models. We construct and study in detail an interesting, and perhaps phenomenologically relevant, model with heavy squarks and light gluinos. The 
lightest supersymmetric particle in the model is the higgsino, so the photino decays into photon + higgsino. The spectrum is explicitly computed in the case of $m_{\tilde{q}}=$ $110 \mathrm{GeV}$ and $m_{\overrightarrow{\mathrm{g}}}=5 \mathrm{GeV}$; the results are typical for the case of a light gluino and heavy squark. Such a scenario cannot be ruled out by the UA1 missing energy events.

We discuss ways to determine all of the parameters of the model and its experimental implications. One interesting consequence is the possibility of detecting the photinos carrying missing momentum in monojet events by surrounding the main detector with a photon detector. The photons would occur in time coincidence with the missing energy event. Since the distance travelled by the photino depends on its mass, observation of photons would not only confirm the presence of photinos but also allow determination of their mass.

Another interesting consequence is a new way to produce a Higgs boson in $\mathrm{e}^{+} \mathrm{e}^{-} \rightarrow \mathrm{Z}^{0} \rightarrow \tilde{\mathrm{h}} \tilde{Z}_{-} \rightarrow \tilde{\mathrm{h}} \tilde{\mathrm{h}} \mathrm{H}^{0}$, with a rate that depends on the $\tilde{Z}_{-}$mass but which could be as large as almost one percent of all $Z^{0}$ decays, even for $m_{\mathrm{H}^{0}}$ up to $\sim \frac{2}{3}$ of $m_{Z}$. The signature is $\mathrm{H}^{0}+$ missing energy, which is quite good for detection.

Finally the higgsino, which is the lightest supersymmetric particle, is a valuable candidate either for hot dark matter, if $m_{\mathrm{h}} \leqslant 100 \mathrm{eV}$, or for cold dark matter, if $m_{\tilde{\mathrm{h}}} \simeq 5 \mathrm{GeV}$.

\section{Appendix A}

\section{EFFECTIVE LOW-ENERGY THEORY FROM MINIMAL $N=1$ SUPERGRAVITY}

In this appendix we will compute the most general low-energy (flat) limit of $N=1$ supergravity with canonical kinetic terms both in the scalar and in the fermionic sector of the theory. The result corresponding to the scalar potential is just an adaptation of a more general super-Higgs mechanism found two years ago by Soni and Weldon [23]; we include it here for completeness and to fix some notations.

The scalar potential of a supersymmetric Yang-Mills theory coupled to minimal $N=1$ supergravity is given by [1]

$$
V=\mathrm{e}^{G}\left(G^{A} G_{A}-3\right)+\frac{1}{2} D^{\alpha} D^{\alpha},
$$

where $G$ is the Kähler potential, taken to be

$$
G=\phi_{A}^{\dagger} \phi^{A}+\log f+\log f^{\dagger},
$$

where $f=f\left(\phi^{A}\right)$ is an analytic function of scalar fields, $\phi^{A}$, the superpotential; $G_{A} \equiv \partial G / \partial \phi^{A}, G^{A} \equiv \partial G / \partial \phi_{A}^{\dagger} ;$ and $D^{\alpha} \equiv g_{\alpha} \phi_{A}^{\dagger}\left(T^{\alpha}\right)_{B}^{A} \phi^{B},\left(T^{\alpha}\right)_{B}^{A}$ being the matrix representation of the gauge generators. We will work, unless explicitly stated, in units of $M=M_{\mathrm{Pl}} / \sqrt{8 \pi}=2.4 \times 10^{18} \mathrm{GeV}$. 
We will divide the scalar fields in two sectors: a hidden sector $Z^{i} \equiv M \zeta^{i}$ which will acquire vacuum expectation values of $O(M)$, and an observable sector $y^{a}$ where all scalar fields will take vacuum expectation values $\ll \mathrm{O}(M)$. The most general $M$-dependence of the superpotential consistent with this separation can be found to be [23]

$$
f\left(\zeta^{i}, y^{a}\right)=M^{2} f_{2}\left(\zeta^{i}\right)+M f_{1}\left(\zeta^{i}\right)+f_{0}\left(\zeta^{i}, y^{a}\right) .
$$

To not break the gauge symmetry at the scale $M$, the fields $\zeta^{i}$ have to be gauge singlets. Although sometimes it may be useful to have several singlets in the hidden sector, and a function $f_{1}\left(\zeta^{i}\right) \neq 0$ in (A.3), we will consider here, without loss of generality in the low-energy theory, the simplest case of only one singlet $\zeta$ in the hidden sector and $f_{1}(\zeta) \equiv 0$. Thus using in (A.1) the superpotential

$$
f\left(\zeta, y^{a}\right)=M^{2} f_{2}(\zeta)+f_{0}\left(\zeta, y^{a}\right),
$$

we get for the scalar potential the following expansion

$$
V=M^{2} V_{2}(\zeta)+V_{00}\left(\zeta, y^{a}\right)+\frac{1}{2} D^{\alpha} D^{\alpha}+\mathrm{O}\left(\frac{1}{M}\right)
$$

where

$$
\begin{aligned}
V_{2}= & E^{2}(\zeta)\left[\left|D f_{2}\right|^{2}-3\left|f_{2}\right|^{2}\right], \\
V_{00}= & V_{2} y_{a}^{\dagger} y^{a}+E^{2}(\zeta)\left\{\left(D f_{2}\right)^{\dagger}\left(D f_{0}\right)+f_{2}^{\dagger}\left(y^{a} \frac{\partial f_{0}^{\dagger}}{\partial y_{a}}-3 f_{0}\right)+\text { h.c. }\right\} \\
& +E^{2}(\zeta)\left[\left|\frac{\partial f_{0}}{\partial y^{a}}\right|^{2}+\left|f_{2}\right|^{2} y_{a}^{\dagger} y^{a}\right],
\end{aligned}
$$

with $E^{2}(\zeta)=\exp \left(\zeta^{\dagger} \zeta\right)$ and $D f_{n}=\partial f_{n} / \partial \zeta+\zeta^{\dagger} f_{n}$.

In the limit $M \rightarrow \infty$ the dominant term in (A.5) will be $M^{2} V_{2}(\zeta)$ so that to find the $\operatorname{VEV} \zeta_{0}$ it is enough to minimize (A.6), $V_{2}^{\prime}\left(\zeta_{0}\right)=0$, and impose the condition for a vanishing cosmological constant, i.e., $D f_{2}\left(\zeta_{0}\right)=\sqrt{3} f_{2}\left(\zeta_{0}\right)$. Now the low-energy effective theory for the observable sector is found by expanding $V_{00}\left(\zeta, y^{a}\right)$ around $\zeta_{0}$

$$
\begin{aligned}
V_{00}\left(\zeta, y^{a}\right) & =V_{00}\left(\zeta_{0}, y^{a}\right)+\sum_{n} \frac{\left(\zeta-\zeta_{0}\right)^{n}}{n !} V_{00}^{(n)}\left(\zeta_{0}, y^{a}\right) \\
& =V_{00}\left(\zeta_{0}, y^{a}\right)+\sum_{n}\left(\frac{\tilde{z}}{M}\right)^{n} \frac{1}{n !} V_{00}^{(n)}\left(\zeta_{0}, y^{a}\right) \underset{M \rightarrow \infty}{\longrightarrow} V_{00}\left(\zeta^{0}, y^{a}\right),
\end{aligned}
$$


where $\tilde{z} \equiv z-\langle z\rangle$ is the physical field in the hidden sector.

If we introduce now a general superpotential $f_{0}\left(\zeta, y^{a}\right)$ as

$$
E\left(\zeta_{0}\right) f_{0}\left(\zeta, y^{a}\right)=g\left(y^{a}\right)+w(\zeta) h\left(y^{a}\right)
$$

where $w\left(\zeta_{0}\right)=0$, we can write the scalar potential (A.8) as

$$
\begin{aligned}
V_{\mathrm{LE}}\left(y^{a}\right)= & \left|\frac{\partial g}{\partial y^{a}}\right|^{2}+m_{3 / 2}^{2} y_{a}^{\dagger} y^{a} \\
& +m_{3 / 2}\left\{\left(\sqrt{3} \zeta_{0}-3\right) g+y^{a} \frac{\partial g}{\partial y^{a}}+\sqrt{3} w^{\prime}\left(\zeta_{0}\right) h+\text { h.c. }\right\}+\frac{1}{2} D^{\alpha} D^{\alpha},
\end{aligned}
$$

where $m_{3 / 2}=\mathrm{e}^{G / 2}=E\left(\zeta_{0}\right) f_{2}\left(\zeta_{0}\right)+\mathrm{O}(1 / M)$ is the gravitino mass. If we decompose the superpotential $g$ into the trilinear, $g^{(3)}$, and quadratic, $g^{(2)}$, terms (we assume there are no singlets in the observable sector) we can cast (A.10) as

$$
\begin{aligned}
V_{\mathrm{LE}}= & \left|\frac{\partial g}{\partial y^{a}}\right|^{2}+m_{3 / 2}^{2} y_{a}^{\dagger} y^{a} \\
& +m_{3 / 2}\left\{A g^{(3)}\left(y^{a}\right)+B g^{(2)}\left(y^{a}\right)+C h\left(y^{a}\right)+\text { h.c. }\right\}+\frac{1}{2} D^{\alpha} D^{\alpha},
\end{aligned}
$$

with $A=\sqrt{3} \zeta_{0}, B=A-1, C=\sqrt{3} w^{\prime}\left(\zeta_{0}\right)$.

We emphasize the difference between this result and the usual case where we assume $w(\zeta) \equiv 1$, i.e., $C=0$. There is no reason for such a choice and the most general low energy effective potential coming from $N=1$ supergravity with canonical kinetic terms is given by (A.11). All the dependence on the super-Higgs mechanism in the hidden sector is contained in the three parameters $A, B$ and $C$ whose values are considered as boundary conditions of the renormalizable low-energy theory.

Finally, the flat limit of the fermion mass matrix

$$
M_{A B}=\mathrm{e}^{G / 2}\left(G_{A B}+G_{A} G_{B}\right)
$$

is easily achieved. Since the super-Higgs mechanism only happens in the $z$-sector (to leading order in $M$ ), the mass term $M_{z z}$ gives rise to a mass term for the goldstino field which disappears in the unitary super-gauge where the goldstino degrees of freedom are eaten by the gravitino. The communication between the hidden and the observable sectors has to disappear for the consistency of the flat limit; in fact, one can show that $M_{z a}=\mathrm{O}(1 / M)$. In the observable sector the mass matrix $M_{a b}\left(\zeta, y^{a}\right)$ 
has to be expanded around the VEV $\zeta_{0}$, as in (A.8), and the result

$$
M_{a b}\left(\zeta, y^{a}\right)=g_{a b}+\mathrm{O}\left(\frac{1}{M}\right),
$$

is identical to a globally supersymmetric theory with superpotential $g\left(y^{a}\right)$.

To conclude the low-energy theory is defined by (A.11) and (A.13). The supertrace mass formula in the effective theory

$$
\operatorname{Str} M^{2}=2(n-1) m_{3 / 2}^{2}
$$

is the same as in the exact theory, before taking the flat limit. This can be understood since the contribution from the $z$-field, $4 m_{3 / 2}^{2}$, exactly cancels the one from the gravitino, $-4 m_{3 / 2}^{2}$, and the soft breakings in (A.11) linear in $m_{3 / 2}$ do not contribute to the supertrace formula. The conservation of the supertrace mass formula, after the limit $M \rightarrow \infty$, is a consistency check since it only contains light $(\ll \mathrm{O}(M))$ degrees of freedom and, thus, no dependence on $M$.

\section{Appendix B}

In this appendix, we provide some of the details required for the phenomenological analysis of our model. The necessary Feynman rules are obtained from ref. [39] (see also ref. [10] for further details). As always, the notation varies among different authors, so we provide a translational table in table 2 relating the notation used here to that of ref. [39].

The mixing of neutral gauginos and higgsinos ("neutralinos") is specified by a $4 \times 4$ matrix which we denote by $Y$. In general, one must use numerical techniques to diagonalize this matrix. Here, we shall make two approximations which will allow us to diagonalize this matrix analytically. First, we take the supersymmetric Higgs mass parameter $m_{4}=0$. This corresponds to the case where the lightest neutralino is a pure higgsino state which is also massless. Second, we take the wino and bino Majorana mass terms (corresponding to the pure weak $\mathrm{SU}(2)$ and $\mathrm{U}(1)$ neutral

TABLE 2

Translation of different notation

\begin{tabular}{cl|ll}
\hline This paper & Ref. [39] & This paper & Ref. [39] \\
\hline$m_{1}^{2}$ & $m_{1}^{2}+\mu^{2}$ & $H_{1}$ & $H_{3}$ \\
$m_{2}^{2}$ & $m_{2}^{2}+\mu^{2}$ & $H_{2}$ & $H_{1}$ \\
$m_{3}^{2}$ & $m_{12}^{2}$ & $H_{3}\left(H_{\text {rad }}\right)$ & $H_{2}$ \\
$m_{4}^{2}$ & $\mu^{2}$ & $M_{1}$ & $M^{\prime}$ \\
$\theta$ & $\beta$ & $M_{2}$ & $M$ \\
\hline
\end{tabular}


states) to be equal, $M_{2}=M_{1}$. Technically, this is not correct except perhaps at the grand unification scale, as can be seen from eq. (4.1). However, if $M_{1}, M_{2} \ll m_{\mathrm{Z}}$, then this approximation is a good one and one finds a pure photino state.

The diagonalizing matrix is denoted by $N$ which satisfies

$$
N^{*} Y N^{-1}=N_{\mathrm{D}}
$$

where $N_{\mathrm{D}}$ is the diagonal neutralino mass matrix. We employ the convention such that the elements of $N_{\mathrm{D}}$ are non-negative; as a result, certain elements of $N$ are purely imaginary. In the approximation described above we find:

$$
N=\left[\begin{array}{cccc}
\cos \theta_{\mathrm{W}} & \sin \theta_{\mathrm{W}} & 0 & 0 \\
-\sin \theta_{\mathrm{W}} \cos \phi_{0} & \cos \theta_{\mathrm{W}} \cos \phi_{0} & \cos \theta \sin \phi_{0} & -\sin \theta \sin \phi_{0} \\
-i \sin \theta_{\mathrm{W}} \sin \phi_{0} & i \cos \theta_{\mathrm{W}} \sin \phi_{0} & -i \cos \theta \cos \phi_{0} & i \sin \theta \cos \phi_{0} \\
0 & 0 & \sin \theta & \cos \theta
\end{array}\right]
$$

where $\tan \theta=v_{2} / v_{1}$. The four Majorana neutralino eigenstates are:

$$
\begin{aligned}
\tilde{\gamma} & =\tilde{B}^{0} \cos \theta_{\mathrm{W}}+\tilde{W}_{3}^{0} \sin \theta_{\mathrm{W}}, \\
\tilde{h} & =\tilde{H}_{1}^{0} \sin \theta+\tilde{H}_{2}^{0} \cos \theta \\
\tilde{Z}_{+} & =\left(-\tilde{B}^{0} \sin \theta_{\mathrm{W}}+\tilde{W}_{3}^{0} \cos \theta_{\mathrm{W}}\right) \cos \phi_{0}+\left(\tilde{H}_{1}^{0} \cos \theta-\tilde{H}_{2}^{0} \sin \theta\right) \sin \phi_{0}, \\
i \tilde{Z}_{-} & =-\left(-\tilde{B}^{0} \sin \theta_{\mathrm{W}}+\tilde{W}_{3}^{0} \cos \theta_{\mathrm{W}}\right) \sin \phi_{0}+\left(\tilde{H}_{1}^{0} \cos \theta-\tilde{H}_{2}^{0} \sin \theta\right) \cos \phi_{0} .
\end{aligned}
$$

The Majorana states $\tilde{Z}_{ \pm}$have mass

$$
M_{\tilde{\mathrm{Z}}_{ \pm}}=\left(m_{\mathrm{Z}}^{2}+\frac{1}{4} M_{\bar{\gamma}}^{2}\right)^{1 / 2} \pm \frac{1}{2} M_{\tilde{\gamma}}
$$

and the mixing angle $\phi_{0}$ is given by:

$$
\tan 2 \phi_{0}=\frac{2 m_{\mathrm{Z}}}{M_{\bar{\gamma}}} .
$$

In the model described in this paper, we have $v_{1} \approx v_{2}$ and $M_{\bar{\gamma}} \ll m_{Z}$. This implies that to first approximation we may take $\phi_{0}=\theta=45^{\circ}$.

If $M_{\tilde{\gamma}}=0$ (implying that $\phi_{0}=45^{\circ}$ ), the $\tilde{Z}_{+}$and $\tilde{Z}_{-}$combine into a neutral Dirac fermion with a mass given by $m_{z}$. Furthermore, when we impose $v_{1}=v_{2}\left(\theta=45^{\circ}\right)$, then the two chargino states are found to become degenerate in mass with a mass given by $m_{\mathrm{w}}$. In some sense this can be regarded as the supersymmetric limit of the model, although this characterization requires some care since supersymmetry 
breaking scalar mass terms (in particular, those corresponding to the Higgs bosons) still remain. It is useful to summarize the properties of the neutral Higgs bosons: $H_{1}, H_{2}$ and $H_{3}$. First, the pseudoscalar $H_{1}^{0}$ is defined by

$$
H_{1}^{0}=\sqrt{2}\left(\operatorname{Im} H_{1}^{1} \sin \theta+\operatorname{Im} H_{2}^{2} \cos \theta\right)
$$

where $\left(H_{1}^{1}, H_{1}^{2}\right)$ and $\left(H_{2}^{1}, H_{2}^{2}\right)$ are the Higgs doublet fields with hypercharge -1 and +1 , respectively. Note that it is $H_{1}^{0}$ which is the supersymmetric partner of the massless higgsino field $\tilde{h}$, as can be seen from eq. (B.3). However, $H_{1}^{0}$ is certainly not massless at tree level, unless $m_{1}=m_{2}=0$ as shown in eq. (3.12). This is why the "supersymmetric limit" described above is somewhat misleading. The scalar fields $H_{2}^{0}$ and $H_{3}^{0}$ are defined by

$$
\begin{aligned}
& H_{2}^{0}=\sqrt{2}\left[\left(\operatorname{Re} H_{1}^{1}-v_{1}\right) \cos \alpha+\left(\operatorname{Re} H_{2}^{2}-v_{2}\right) \sin \alpha\right], \\
& H_{3}^{0}=\sqrt{2}\left[-\left(\operatorname{Re} H_{1}^{1}-v_{1}\right) \sin \alpha+\left(\operatorname{Re} H_{2}^{2}-v_{2}\right) \cos \alpha\right],
\end{aligned}
$$

which defines the mixing angle $\alpha$. A few useful formulas are given below:

$$
\begin{aligned}
\tan 2 \alpha & =\frac{\tan 2 \theta\left(m_{H_{2}}^{2}+m_{H_{3}}^{2}\right)}{m_{H_{1}}^{2}-m_{\mathrm{Z}}^{2}}, \\
\sin ^{2}(\theta-\alpha) & =\frac{m_{H_{2}}^{2}\left(m_{H_{2}}^{2}-m_{\mathrm{Z}}^{2}\right)}{\left(m_{H_{2}}^{2}-m_{H_{3}}^{2}\right)\left(m_{H_{2}}^{2}+m_{H_{3}}^{2}-m_{\mathrm{Z}}^{2}\right)}, \\
\sin ^{2}(\theta+\alpha) & =\frac{m_{H_{3}}^{2}\left(m_{H_{2}}^{2}-m_{\mathrm{Z}}^{2}\right)}{m_{\mathrm{Z}}^{2}\left(m_{H_{2}}^{2}-m_{H_{3}}^{2}\right)}, \\
m_{H_{2}}^{2}+m_{H_{3}}^{2} & =m_{H_{1}}^{2}+m_{\mathrm{Z}}^{2},
\end{aligned}
$$

where $0 \leqslant m_{H_{3}} \leqslant m_{Z}$ and $m_{H_{2}} \geqslant m_{Z}$. In the above formulas, the Higgs masses are tree-level masses only. It is conventional to choose phases such that $v_{1}$ and $v_{2}$ are positive and so $0 \leqslant \theta \leqslant 90^{\circ}$. In this case, it follows from the conventions above (i.e. from eqs. (B.7), (B.8) and the requirement that $m_{H_{3}}<m_{H_{2}}$ ) that $-90^{\circ} \leqslant \alpha \leqslant 0$. In terms of the supersymmetry breaking Higgs mass parameters, the neutral Higgs masses are given by eqs. (3.12), (3.13). Note that in the limit of $v_{1}=v_{2}, \theta=45^{\circ}$ and using eq. (B.9) and the phase conventions discussed above, it follows that $\alpha=-45^{\circ}$. From eq. (B.10) and (B.11), it follows that $m_{H_{3}}=0$ as noted below eq. (3.14) ${ }^{\star}$. Note

* It is curious that it is $\mathrm{H}_{3}$ which is massless (at tree level), whereas $\mathrm{H}_{1}$ is massive; although it is $\mathrm{H}_{1}$ which is the supersymmetric partner of the massless higgsino, $\tilde{\mathrm{h}}$. 
TABLE 3

Feynman rules for $\mathrm{qq} \tilde{\chi}^{0}$ vertices

\begin{tabular}{ccc}
\hline & $\tilde{\chi}^{0} \equiv \tilde{\gamma}$ & $\tilde{\chi}^{0} \approx \tilde{\mathrm{h}}$ \\
\hline$\tilde{\mathrm{u}}_{\mathrm{L}} \mathrm{u} \tilde{\chi}^{0}$ & $-\sqrt{\frac{1}{2}} i e e_{\mathrm{u}}\left(1+\gamma_{5}\right)$ & $\frac{-i g_{2} m_{\mathrm{u}} \cot \theta}{2 \sqrt{2} m_{\mathrm{W}}}\left(1-\gamma_{5}\right)$ \\
$\tilde{\mathrm{u}}_{\mathrm{R}} \mathrm{u} \tilde{\chi}^{0}$ & $\sqrt{\frac{1}{2}} i e e_{\mathrm{u}}\left(1-\gamma_{\mathrm{S}}\right)$ & $\frac{-i g_{2} m_{\mathrm{u}} \cot \theta}{2 \sqrt{2} m_{\mathrm{W}}}\left(1+\gamma_{5}\right)$ \\
$\tilde{\mathrm{d}}_{\mathrm{L}} \mathrm{d} \tilde{\chi}^{0}$ & $-\sqrt{\frac{1}{2}}-i e e_{\mathrm{d}}\left(1+\gamma_{5}\right)$ & $\frac{-i g_{2} m_{\mathrm{d}} \tan \theta}{2 \sqrt{2} m_{\mathrm{W}}}\left(1-\gamma_{5}\right)$ \\
$\tilde{\mathrm{d}}_{\mathrm{R}} \mathrm{d} \tilde{\chi}^{0}$ & $\sqrt{\frac{1}{2} i e e_{\mathrm{d}}\left(1-\gamma_{5}\right)}$ & $\frac{-i g_{2} m_{\mathrm{d}} \tan \theta}{2 \sqrt{2} m_{\mathrm{W}}}\left(1+\gamma_{5}\right)$ \\
\hline
\end{tabular}

For definiteness, we take the momentum of $\tilde{q}$ into the vertex and the momentum of $q$ out of the vertex. The left-handed projection operator is $\frac{1}{2}\left(1-\gamma_{s}\right)$. The quark charge (in units of $e$ ) is denoted by $e_{\mathrm{q}}$. where $\mathrm{q}=\mathrm{u}, \mathrm{d}$.

that although $\mathrm{H}_{3}$ gains a non-zero mass due to radiative corrections, factors such as $\sin (\theta+\alpha)$ which appear in Feynman rules are to be computed using tree-level masses in eq. (B.11).

We are now in the position to state the Feynman rules relevant to the analysis here; they are given in table 3.

As an application of these rules, we compute the decay rate for $\tilde{\gamma} \rightarrow \tilde{\mathrm{h}} \gamma$. The possible diagrams are shown in figs. $1 \mathrm{a}, \mathrm{b}$. Consider first the diagrams involving the $q-\tilde{q}$ loop shown in fig. 1a. The amplitude has contributions from fig. 1a(A)-(D) which we write as:

$$
M=\sum_{\substack{i=\mathrm{L}, \mathrm{R} \\ j=\mathrm{A}, \mathrm{B}, \mathrm{C}, \mathrm{D}}} M_{i j}
$$

where $i=\mathrm{L}, \mathrm{R}$ refers to either $\tilde{\mathrm{q}}_{\mathrm{L}}$ or $\tilde{\mathrm{q}}_{\mathrm{R}}$ inside the loop. Note that the second two diagrams are obtained from the first two by reversing the circulation of the momentum flow inside the loop. One can show that $M_{\mathrm{C}}$ (and $M_{\mathrm{D}}$ ) are obtained from $M_{\mathrm{A}}$ (and $M_{\mathrm{B}}$ ) by changing $\gamma_{5}$ to $-\gamma_{5}$ and multiplying the resulting amplitude by -1 . The factor of -1 is due to Fermi statistics and arises because reversing the momentum flow is equivalent to the exchange of the two outgoing fermion lines. Thus, we obtain:

$$
\begin{array}{ll}
M_{\mathrm{LA}}=M_{\mathrm{RC}}, & \mathrm{L} \leftrightarrow \mathrm{R}, \\
M_{\mathrm{LB}}=M_{\mathrm{RD}}, & \mathrm{L} \leftrightarrow \mathrm{R} .
\end{array}
$$

Note that a crucial ingredient which led to this result is the relative minus sign 
which occurs in the rule for $\tilde{\mathrm{q}} q \tilde{\gamma}$ between $\tilde{\mathrm{q}}_{L}$ and $\tilde{\mathrm{q}}_{\mathrm{R}}$ but does not occur in the rule for $\overline{\mathrm{q}} \tilde{\mathrm{h}}$ (see table 3). This is to be contrasted with that occurring in the calculation of the $\tilde{\gamma} \tilde{\gamma} \gamma$ vertex. In this case, two minus signs would result when comparing $\tilde{\mathrm{q}}_{\mathrm{L}}$ and $\tilde{\mathrm{q}}_{\mathrm{R}}$ in the loop; as a result, we would find $M_{\mathrm{LA}}=-M_{\mathrm{RC}}, M_{\mathrm{LB}}=-M_{\mathrm{RD}}$, and $\mathrm{L} \leftrightarrow \mathrm{R}$. Then, in the limit of degenerate $\tilde{\mathrm{q}}_{\mathrm{L}}$ and $\tilde{\mathrm{q}}_{\mathrm{R}}$ (the $P$ and $C$ conserving limit), the total amplitude $M$ (eq. (B.13)) would vanish. This is just the supersymmetric analog of Furry's theorem and has been previously noted in the calculation of $\tilde{\mathrm{g}} \rightarrow \mathrm{g} \tilde{\gamma}$ [38]. In the case of $\tilde{\gamma} \rightarrow \tilde{\mathrm{h}} \gamma$, we know that the amplitude must not vanish in the limit of $\tilde{\mathrm{q}}_{L}$ and $\tilde{\mathrm{q}}_{R}$ as we have demonstrated via eqs. (B.13) and (B.14) since the supersymmetric analog, the $\mathrm{H}^{0} \gamma \gamma$ vertex, conserves $P$ and $C$ and certainly does not vanish at one loop.

In the discussion above, we have treated $\tilde{q}_{L}$ and $\tilde{q}_{R}$ as the mass eigenstates. Although this is usually a good approximation, it is likely to be inaccurate in the case of the t-quark. Schematically, if the Feynman rules for $\tilde{\mathrm{q}}_{\mathrm{L}} \mathrm{q} \tilde{\mathrm{h}}$ and $\tilde{\mathrm{q}}_{\mathrm{R}} \mathrm{q} \tilde{\mathrm{h}}$ are given by $A_{\mathrm{L}}$ and $A_{\mathrm{R}}$ respectively, then the Feynman rules involving the mass eigenstates $\tilde{\mathrm{q}}_{1}$ and $\tilde{\mathrm{q}}_{2}$ are given by:

$$
\begin{aligned}
& A\left(\tilde{\mathrm{q}}_{1} \mathrm{q} \tilde{\mathrm{h}}\right)=A_{\mathrm{L}} \cos \theta_{\mathrm{q}}+A_{\mathrm{R}} \sin \theta_{\mathrm{q}}, \\
& A\left(\tilde{\mathrm{q}}_{2} \mathrm{q} \tilde{\mathrm{h}}\right)=-A_{\mathrm{L}} \sin \theta_{\mathrm{q}}+A_{\mathrm{R}} \cos \theta_{\mathrm{q}},
\end{aligned}
$$

where $\theta_{\mathrm{q}}$ is the mixing angle between the interaction and mass eigenstates. Using eq. (B.15) and the rules of table 3 , we have computed the diagrams shown in fig. 1. Using the recipe discussed above in eq. (B.14), we end up with:

$$
\begin{aligned}
M= & \frac{3 e_{\mathrm{q}}^{2} e^{2} g_{2} m_{\mathrm{q}}^{2} \cot \theta}{16 \pi^{2} m_{\mathrm{W}} M_{\tilde{\gamma}}^{2}} i \bar{u}\left(k_{1}\right) \gamma_{5} \sigma^{\mu \nu} \varepsilon_{\mu}^{*}\left(k_{1}\right) k_{2 \nu} u(p) \\
& \times\left\{\int_{0}^{1} \frac{\mathrm{d} x}{1-x}\left(\log X_{1}+\log X_{2}\right)\right. \\
& \left.\quad-\frac{M_{\tilde{\gamma}}}{m_{\mathrm{q}}} \sin 2 \theta_{\mathrm{q}} \int_{0}^{1} \mathrm{~d} x\left(\frac{\log X_{1}}{1-X_{1}}-\frac{\log X_{2}}{1-X_{2}}\right)\right\},
\end{aligned}
$$

where

$$
X_{i}=1-\frac{M_{\tilde{\gamma}}^{2} x(1-x)}{m_{\mathrm{q}}^{2} x+M_{\hat{\mathrm{q}}_{i}}^{2}(1-x)} .
$$

Note that a factor of 3 has been inserted due to the color of the internal loop. The factor of $\cot \theta$ appears if the quark in the loop is an up-type quark. If the quark is a down-type, we replace $\cot \theta$ with $\tan \theta$. The integrals can be expressed in terms of 
dilogarithms, but it is more useful to consider various limiting cases. First, we note that in the supergravity model considered in this paper, we have $\theta_{\mathrm{q}}=0$ unless $\mathrm{q}$ is the t-quark. But in the case of the t-quark, we may drop the second term in eq. (B.16) since $M_{\tilde{\gamma}} \ll m_{\mathrm{t}}$. The result can be further simplified if the approximation $M_{\tilde{\gamma}} \ll m_{t}, M_{\tilde{i}_{i}}$ is applied to the first term. Clearly, the t-quark loop dominates, so we neglect all other quark flavors and find:

$$
\begin{aligned}
M= & \frac{-3 e_{\mathrm{t}}^{2} e^{2} g_{2} m_{t}^{2} \cot \theta}{16 \pi^{2} m_{\mathrm{W}}} i \bar{u}\left(k_{1}\right) \gamma_{5} \sigma^{\mu \nu} \varepsilon_{\mu}^{*}\left(k_{1}\right) k_{2 \nu} u(p) \\
& \times \sum_{i=1}^{2} \frac{1}{m_{\mathrm{t}}^{2}-M_{\mathrm{i}_{i}}^{2}}\left(1-\frac{M_{\mathrm{i}_{i}}^{2}}{m_{\mathrm{t}}^{2}-M_{\mathrm{t}_{i}}^{2}} \log \frac{m_{\mathrm{t}}^{2}}{M_{\mathrm{i}_{i}}^{2}}\right) .
\end{aligned}
$$

The decay rates obtained from these matrix elements have been discussed in sect. 5 .

In order to check these results, it is instructive to check the supersymmetric limit, which relates the $\tilde{\mathrm{h}} \gamma \tilde{\gamma}$ and $\mathrm{H} \gamma \gamma$ vertices. In this comparison, it is appropriate to take $M_{\tilde{\gamma}}=0$ and $M_{\tilde{\mathrm{h}}}=m_{\mathrm{H}} \neq 0$. So, we need to consider the supersymmetric decay process $\tilde{\mathrm{h}} \rightarrow \mathrm{h} \tilde{\gamma}$. It is easy to see that for this case, one obtains eq. (B.16) with the replacement $M_{\tilde{\gamma}}$ by $M_{\overline{\mathrm{h}}}$. Finally, we put $M_{\mathrm{q}}=M_{\tilde{\mathrm{q}}_{1}}$ and $\theta_{\mathrm{q}}=0$ as is appropriate for the supersymmetric limit. Using

$$
I \equiv \int_{0}^{1} \frac{\mathrm{d} x}{1-x} \log \left(1-\frac{m_{\mathrm{H}}^{2}}{m_{\mathrm{q}}^{2}} x(1-x)\right)=-2\left[\sin ^{-1}\left(\frac{m_{\mathrm{H}}}{2 m_{\mathrm{q}}}\right)\right]^{2},
$$

we see that in the supersymmetric limit, the effective $\tilde{h} \rightarrow \gamma \tilde{\gamma}$ decay vertex takes the form

$$
M(\tilde{\mathrm{h}} \rightarrow \gamma \tilde{\gamma})=\frac{g_{2} \alpha e_{\mathrm{q}}^{2} N_{c} \cot \theta m_{\mathrm{q}}^{2}}{2 \pi m_{\mathrm{W}} m_{\mathrm{H}}^{2}}\left[\sin ^{-1}\left(\frac{m_{\mathrm{H}}}{2 m_{\mathrm{q}}}\right)\right]^{2} \overline{\tilde{\gamma}} \gamma_{5} \sigma^{\mu \nu} \tilde{\chi} F_{\mu \nu},
$$

where $N_{c}=3$ is the number of colors. Note that in translating from eq. (B.20) to the appropriate Feynman rule, we replace $F^{\mu \nu} \rightarrow 2 i \varepsilon^{* \mu} k^{\nu}$.

Next, consider the decay $\mathrm{H}_{1}^{0} \rightarrow \gamma \gamma$. Recall that $\mathrm{H}_{1}^{0}$ is the pseudoscalar. A straightforward computation yields:

$$
M\left(\mathrm{H}_{1}^{0} \rightarrow \gamma \gamma\right)=\frac{g_{2} \alpha e_{\mathrm{q}}^{2} N_{c} \cot \theta m_{\mathrm{q}}^{2}}{\pi m_{\mathrm{W}} m_{\mathrm{H}}^{2}}\left[\sin ^{-1}\left(\frac{m_{\mathrm{H}}}{2 m_{\mathrm{q}}}\right)\right]^{2} H_{1}^{0} F_{\mu \nu} \tilde{F}^{\mu \nu},
$$

where $\tilde{F}_{\mu \nu}=\frac{1}{2} \varepsilon_{\mu \nu \alpha \beta} F^{\alpha \beta}$ which indeed confirms our previous computation, and also confirms the observation that the supersymmetric partner of $\tilde{h}$ is the pseudoscalar $\mathrm{H}_{1}^{0}$. The amplitude for the scalar Higgs decay into two photons differs in three ways 
from that of eq. (B.21). First, $H_{1}^{0} F \tilde{F}$ is relaced by $H_{i}^{0} F F$ as required by $C P$-invariance of the interaction. Second, the integral $I$ in eq. (B.19) is replaced by:

$$
l=1+\frac{x-1}{x}\left[\sin ^{-1} \sqrt{x}\right]^{2}
$$

with $x \equiv m_{\mathrm{H}}^{2} / 4 m_{\mathrm{q}}^{2}$. Finally, the factor of $\cot \theta$ is replaced by a different combination of factors involving both $\theta$ and $\alpha$, appropriate to the $\mathrm{H}_{i}^{0} \mathrm{q} \overline{\mathrm{q}}$ vertex. We have displayed these differences to accentuate the fact that $\tilde{h}$ is the superpartner of the pseudoscalar $\mathrm{H}_{1}^{0}$ and not the scalar Higgs $\mathrm{H}_{2}^{0}$ or $\mathrm{H}_{3}^{0}$.

So far, we have neglected the diagrams of fig. $1 \mathrm{~b}$. We shall argue here that they will not be important in the context of the model we have pursued. First, consider the decay $\mathrm{H}_{1}^{0} \rightarrow \gamma \gamma$. Because $\mathrm{H}_{1}^{0}$ is a pseudoscalar, there are no contributing diagrams involving internal $\mathrm{W}$ or charged Higgs boson loops. This follows from the $C P$-invariance of the relevant interactions. (Of course, for the scalar Higgs decay into $\gamma \gamma$, these additional diagrams do contribute and are important.) Thus, we conclude that in the supersymmetric limit, the contribution of the diagrams of fig. $1 \mathrm{~b}$ to the $\tilde{\mathrm{h}} \gamma \tilde{\gamma}$ vertex must vanish. But, the model discussed in this paper is very close to the supersymmetric limit in that $\theta \simeq 45^{\circ}, \alpha \simeq-45^{\circ}, M_{\tilde{\gamma}} \ll m_{\mathrm{Z}}, M_{\tilde{Z}_{+}} \approx$ $M_{\tilde{\mathrm{Z}}_{-}} \approx m_{\mathrm{Z}}$ and $M_{\tilde{\mathrm{h}}_{1}^{+}} \approx M_{\tilde{\mathrm{h}}^{+}} \approx M_{\mathrm{W}}$. The fact that the scalar quarks are split in mass from their quark partners is not relevant in the evaluation of the diagrams of fig. $1 \mathrm{~b}$. We conclude that in the model considered in this paper, the diagrams of fig. 1a must dominate the $\tilde{\gamma}$ decay rate. Explicit calculations of the diagrams shown in fig. $1 \mathrm{~b}$ will be presented elsewhere.

\section{Appendix C}

We have been examining a model which contains a very light higgsino $\tilde{h}$ which we have taken to be massless for convenience. In addition, we have seen that there are two neutralino states $\tilde{Z}_{ \pm}$with masses given by eq. (B.4). Since $M_{\tilde{Z}}<m_{Z}$, the decay $Z^{0} \rightarrow \tilde{Z}_{-}+\tilde{h}$ is kinematically allowed. The decay rate is easily computed. We normalize our rates to

$$
\Gamma\left(\mathrm{Z}^{0} \rightarrow \nu \bar{\nu}\right)=\frac{g_{2}^{2} m_{\mathrm{Z}}}{96 \pi \cos ^{2} \theta_{\mathrm{W}}}
$$

We find:

$$
\frac{\Gamma\left(\mathrm{Z}^{0} \rightarrow \tilde{\mathrm{Z}}_{-}+\tilde{\mathrm{h}}\right)}{\Gamma\left(\mathrm{Z}^{0} \rightarrow \nu \bar{\nu}\right)}=2 \cos ^{2} \phi_{0} \sin ^{2} 2 \theta\left(1-M_{\mathrm{Z}_{-}}^{2} / m_{\mathrm{Z}}^{2}\right)^{2}\left(1+M_{\mathrm{Z}_{-}}^{2} / 2 m_{Z}^{2}\right)
$$

In the model discussed in this paper, $\sin 2 \theta \approx 1$ and $2 \cos ^{2} \phi_{0} \approx 1$. So, the major 
suppression here is due to phase space, since by eq. (B.4), $\tilde{M}_{\mathrm{Z}_{-}} \approx M_{\mathrm{Z}}-\frac{1}{2} M_{\tilde{\gamma}}$ (for $M_{\tilde{\gamma}} \ll m_{\mathrm{Z}}$ ). In more general models with a light higgsino but with a photino mass which is not particularly small, we see that $\mathrm{BR}\left(\mathrm{Z}^{0} \rightarrow \tilde{Z}_{-} \tilde{\mathrm{h}}\right)$ could be substantial.

In order to observe this particular decay of the $Z^{0}$, we must discuss the decay signature of the $\tilde{Z}_{-}$. If the gluino is light as advocated in this paper, then the dominant decay of the $\tilde{Z}_{-}$will be:

$$
\tilde{\mathrm{Z}}_{-} \rightarrow \mathrm{q} \overline{\mathrm{q}} \tilde{\mathrm{g}} .
$$

This decay occurs via an intermediate $\tilde{\mathrm{q}}$ and is analogous to $\tilde{\mathrm{g}} \rightarrow \mathrm{q} \overline{\mathrm{q}} \tilde{\gamma}$. The computation is very similar and we summarize our results here. Neglecting all the final state masses,

$$
\Gamma\left(\tilde{\mathrm{Z}}_{-} \rightarrow \mathrm{q} \overline{\mathrm{q}} \tilde{\mathrm{g}}\right)=\frac{\alpha_{\mathrm{s}} g_{2}^{2} M_{\overline{\mathrm{Z}}_{-}}^{5} \sin ^{2} \phi_{0}}{48 \pi^{2} \cos ^{2} \theta_{\mathrm{W}} M_{\tilde{\mathrm{q}}}^{4}}\left[\left(T_{3 \mathrm{q}}-e_{\mathrm{q}} \sin ^{2} \theta_{\mathrm{w}}\right)^{2}+e_{\mathrm{q}}^{2} \sin ^{4} \theta_{\mathrm{w}}\right],
$$

where we have taken $\tilde{\mathrm{q}}_{L}$ and $\tilde{\mathrm{q}}_{\mathrm{R}}$ to be degenerate in mass. In deriving the above formula, we sum over the first two generations and the b-quark. Taking $\sin ^{2} \phi_{0} \approx \frac{1}{2}$, and $M_{\overline{\mathbf{Z}}_{-}} \approx m_{\mathrm{Z}}$,

$$
\frac{\sum_{\mathrm{q}} \Gamma\left(\tilde{\mathrm{Z}}_{-} \rightarrow \mathrm{q} \overline{\mathrm{q}} \tilde{\mathrm{g}}\right)}{\Gamma\left(\mathrm{Z}^{0} \rightarrow \nu \bar{\nu}\right)}=\frac{0.84 \alpha_{\mathrm{s}}}{\pi}\left(\frac{M_{\tilde{\mathrm{Z}}_{-}}}{M_{\overline{\mathrm{q}}}}\right)^{4} .
$$

For example, if $M_{\tilde{\mathrm{q}}} \approx M_{\tilde{\mathrm{Z}}_{-}}$, we obtain about $3 \times 10^{-2}$ for the above ratio implying a

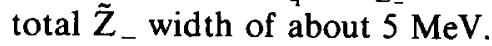

The $q \bar{q} \tilde{g}$ final state is not the ideal one to identify the $\tilde{Z}_{-}$. So, let us consider an alternative possibility. The vertices given in table 4 suggest that we consider:

$$
\tilde{\mathrm{Z}}_{-} \rightarrow \tilde{\mathrm{h}} \mathrm{H}_{3}^{0} .
$$

Since $\mathrm{H}_{3}^{0}$ is light in our model and $\tilde{h}$ is taken to be massless, there will be no phase space suppression. We find:

$$
\Gamma\left(\tilde{\mathrm{Z}}_{-} \rightarrow \tilde{\mathrm{h}} \mathrm{H}_{3}^{0}\right)=\frac{g_{2}^{2} \sin ^{2} \phi_{0} \cos ^{2}(\theta-\alpha)}{64 \pi \cos ^{2} \theta_{\mathrm{w}}} \frac{\left(M_{\mathrm{Z}_{-}}^{2}-M_{\mathrm{H}_{3}}^{2}\right)^{2}}{M_{\mathrm{Z}_{-}}^{3}} .
$$

Neglecting the mass of $\mathrm{H}_{3}$, taking $M_{\hat{\mathrm{Z}}_{-}} \approx M_{\mathrm{Z}}$, and normalizing to $\Gamma\left(\mathrm{Z}^{0} \rightarrow \nu \bar{\nu}\right) \approx$ $150 \mathrm{MeV}$ for convenience, we find

$$
\frac{\Gamma\left(\tilde{\mathrm{Z}}_{-} \rightarrow \tilde{\mathrm{h}} \mathrm{H}_{3}^{0}\right)}{\Gamma\left(\mathrm{Z}^{0} \rightarrow \nu \bar{\nu}\right)} \approx \frac{3}{2} \sin ^{2} \phi_{0} \cos ^{2}(\theta-\alpha) .
$$


TABLE 4

Feynman rules involving $\tilde{Z}_{ \pm}$

\begin{tabular}{|c|c|c|}
\hline & $\tilde{\mathbf{Z}}=\tilde{\mathbf{Z}}_{+}$ & $\tilde{Z}=\tilde{Z}_{-}$ \\
\hline \multirow{2}{*}{$\mathrm{Z}^{0} \tilde{\mathrm{Z}} \overline{\mathrm{h}}$} & $i g_{2} \sin \phi_{0} \sin 2 \theta$ & $g_{2} \cos \phi_{0} \sin 2 \theta \gamma^{\mu}$ \\
\hline & $2 \cos \theta_{W} \gamma^{\gamma} \gamma_{5}$ & $2 \cos \theta_{\mathrm{W}}$ \\
\hline \multirow{2}{*}{$\mathrm{H}_{1}^{0} \tilde{\mathrm{Z}} \overline{\mathrm{h}}$} & $-g_{2} \cos \phi_{0} \cos 2 \theta$ & $-i g_{2} \sin \phi_{0} \cos 2 \theta$ \\
\hline & $2 \cos \theta_{W} \gamma_{5}$ & $2 \cos \theta_{w}$ \\
\hline \multirow{2}{*}{$\mathrm{H}_{2}^{0} \tilde{\mathrm{Z}} \tilde{\mathrm{h}}$} & $-i g_{2} \cos \phi_{0} \sin (\theta-\alpha)$ & $g_{2} \sin \phi_{0} \sin (\theta-\alpha)$ \\
\hline & $2 \cos \theta_{\mathrm{W}}$ & $2 \cos \theta_{\mathrm{W}} \gamma_{5}$ \\
\hline \multirow{2}{*}{$\mathrm{H}_{3}^{0} \tilde{Z} \tilde{\mathrm{h}}$} & $i g_{2} \cos \phi_{0} \cos (\theta-\alpha)$ & $-g_{2} \sin \phi_{0} \cos (\theta-\alpha)$ \\
\hline & $2 \cos \theta_{\mathrm{W}}$ & $2 \cos \theta_{\mathrm{w}}$ \\
\hline \multirow{2}{*}{$\tilde{\mathrm{Z}} \mathrm{q} \tilde{\mathrm{q}}_{\mathrm{L}}$} & $-i g_{2} \cos \phi_{0}\left(T_{3 \mathrm{q}}-e_{\mathrm{q}} \sin ^{2} \theta_{\mathrm{W}}\right)$ & $g_{2} \sin \phi_{0}\left(T_{3 \mathrm{q}}-e_{\mathrm{q}} \sin ^{2} \theta_{\mathrm{W}}\right)$ \\
\hline & $\sqrt{2} \cos \theta_{\mathrm{w}}$ & $\sqrt{2} \cos \theta_{\mathrm{w}}\left(1+\gamma_{5}\right)$ \\
\hline$\tilde{Z}_{q} \tilde{q}_{R}$ & $\frac{-i g_{2} \cos \phi_{0} e_{\mathrm{q}} \sin ^{2} \theta_{\mathrm{w}}}{\sqrt{2} \cos \theta_{\mathrm{w}}}\left(1-\gamma_{5}\right)$ & $\frac{-g_{2} \sin \phi_{0} e_{\mathrm{q}} \sin ^{2} \theta_{\mathrm{w}}}{\sqrt{2} \cos \theta_{\mathrm{w}}}\left(1-\gamma_{5}\right)$ \\
\hline
\end{tabular}

For definiteness, we take the momentum of $\tilde{\mathrm{Z}}_{ \pm}$into the vertex and the momentum of $\tilde{\mathrm{h}}$ (or $\mathrm{q}$ ) out of the vertex. Note that the factor of $i$ in the definition of $\tilde{Z}_{-}$(eq. (B.3)) is responsible for some of the important differences between the rules for $\tilde{Z}_{+}$and $\tilde{Z}_{\ldots}$. We take $e_{\mathrm{u}}=\frac{2}{3}, e_{\mathrm{d}}=-\frac{1}{3}, T_{3 \mathrm{u}}=\frac{1}{2}, T_{3 \mathrm{~d}}=-\frac{1}{2}$.

Comparing this to $\Gamma\left(\tilde{Z}_{-} \rightarrow q \bar{q} \tilde{\mathrm{g}}\right)$, it appears that the $\tilde{Z}_{-} \rightarrow \tilde{\mathrm{h}} \mathrm{H}_{3}^{0}$ decay mode should dominate! However, using eq. (B.9), we find:

$$
\cos ^{2}(\theta-\alpha)=\frac{M_{H_{3}}^{2}\left(M_{\mathrm{Z}}^{2}-M_{\mathrm{H}_{3}}^{2}\right)}{\left(M_{\mathrm{H}_{2}}^{2}-M_{\mathrm{H}_{3}}^{2}\right)\left(M_{\mathrm{H}_{2}}^{2}+M_{\mathrm{H}_{3}}^{2}-M_{\mathrm{Z}}^{2}\right)},
$$

where, it must be emphasized, tree-level masses must be used in this equation. Since the tree-level value for $M_{\mathrm{H}_{3}}$ is rather small (and, in fact, vanishes in the limit of $\left.v_{1}=v_{2}\right)$, we see that $\cos ^{2}(\theta-\alpha)$ can be quite small. As noted below eq. (B.11), the model considered in this paper is rather close to the values $\theta=-\alpha=45^{\circ}$ which would imply $\cos (\theta-\alpha)=0$. However, the above conclusions need not hold in more general models with a light higgsino [35].

\section{References}

[1] J. Ellis. in Proc. Yukon Advanced Study Institute in the Quark structure of matter, ed. N. Ishur. G. Kedland, P. J. O'Donnell, (World Scientific, Singapore, 1985) p. 256:

D.V. Nanopoulos, CERN preprint TH.3995 (1984):

H.P. Nilles, Phys. Reports C110 (1984) 2;

P. Nath. R. Arnowitt and A.H. Chamseddine, Applied $N=1$ supergravity, ICTP Series in Theoretical Physics, vol. I (World Scientific, Singapore, 1985)

[2] R. Barbieri, S. Ferrara and C.A. Savoy, Phys. Lett. 119B (1982) 343; 
L. Hall, J. Lykken and S. Weinberg, Phys. Rev. D27 (1983) 346;

H.P. Nilles, M. Srednicki and D. Wyler, Phys. Lett. 120B (1983) 346

[3] K. Inoue, A. Kakuto, H. Komatsu and S. Takeshita, Prog. Theor. Phys. 67 (1982) 1889, 68 (1982) 927, 71 (1984) 413

[4] L. Alvarez-Gaumé, J. Polchinski and M. Wise, Nucl. Phys. B221 (1983) 495

[5] J. Ellis, J. Hagelin, D.V. Nanopoulos and K. Tamvakis, Phys. Lett. 125B (1983) 275

[6] M. Claudson, L. Hall and I. Hinchliffe, Nucl. Phys. B228 (1983) 501

[7] L. Ibáñez and C. López, Phys. Lett. 126B (1983) 54; Nucl. Phys. B233 (1984) 511

[8] C. Kounnas, A.B. Lahanas, D.V. Nanopoulos and M. Quiros, Phys. Lett. 132B (1983) 95: Nucl. Phys. B236 (1984) 438

[9] UAl Collaboration, G. Arnison et al., Phys. Lett. 147B (1984) 493

[10] H.E. Haber and G.L. Kane, Phys. Reports C117 (1985) 75

[11] UAl Collaboration, G. Arnison et al., Phys. Lett. 139B (1984) 115

[12] J. Rohlf, invited talk at the 1985 Division of Particles and Fields Conference, Eugene, Oregon, August 1985:

C. Rubbia, invited talk at the 1985 Lepton-Photon Conference. Kyoto, Japan, August 1985

[13] R.M. Barnett, H.E. Haber and G.L. Kane, Nucl. Phys. B267 (1986) 625

[14] E. Reya and D.P. Roy, Dortmund preprint DO-TH 85/23 (1985)

[15] A. de Rújula and R. Petronzio, Nucl. Phys. B261 (1985) 587

[16] See refs. $[17,18,19,15,13]$

[17] M.J. Herrero, L. E. Ibáñez, C. López and F.J. Yndurain, Phys. Lett. 132B (1983) 199; 145B (1984) 430

[18] V. Barger, K. Hagiwara, W. Keung and J. Woodside, Phys. Rev. Lett. 53 (1984) 641

[19] R. M. Barnett, H.E. Haber and G.L. Kane, Phys. Rev. Lett. 54 (1985) 1983

[20] L.E. Ibáñez, C. López and C. Muñoz, Nucl. Phys. B256 (1985) 252

[21] K. Enqvist, D.V. Nanopoulos and A.B. Lahanas, Phys. Lett. 155B (1985) 83

[22] J. Ellis, A.B. Lahanas, D.V. Nanopoulos and K. Tamvakis, Phys. Lett. 134B (1984) 429;

J. Ellis, C. Kounnas and D.V. Nanopoulos, Nucl. Phys. B241 (1984) 406, B247 (1984) 373; Phys. Lett. 143B (1984) 410;

J. Ellis, K. Enqvist and D.V. Nanopoulos, Phys. Lett. 147B (1984) 99, 151 B (1985) 357;

N. Dragon, U. Ellwanger, and M.G. Schmidt, Phys. Lett. 145B (1984) 192; 154B (1985) 373; Nucl. Phys. B255 (1985) 549; CERN-TH 4129/85

[23] S.K. Soni and H.A. Weldon, Phys. Lett. 126B (1983) 215

[24] J.M. Frére, D.R.T. Jones, and S. Raby, Nucl. Phys. B222 (1983) 11

[25] J.E. Kim, A. Masiero and D.V. Nanopoulos, Phys. Lett. 139B (1984) 346

[26] J. Ellis, K. Enqvist, D.V. Nanopoulos and K. Tamvakis, Phys. Lett. 156B (1985) 189

[27] B. Grinstein, Nucl. Phys. B206 (1982) 387;

A. Masiero, D.V. Nanopoulos, K. Tamvakis and T. Yanagida, Phys. Lett. 115B (1982) 380;

C. Kounnas, D.V. Nanopoulos, M. Quirós and M. Srednicki, Phys. Lett. 127B (1983) 82

[28] J.-M. Frére and G.L. Kane, Nucl. Phys. B223 (1983) 331;

J. Ellis, J.S. Hagelin, D.V. Nanopoulos and M. Srednicki, Phys. Lett. 127B (1983) 233

[29] J. Ellis, J.S. Hagelin, D.V. Nanopoulos, K. Olive, and M. Srednicki, Nucl. Phys. B238 (1984) 453

[30] J. Ellis, C. Kounnas and D.V. Nanopoulos, Nucl. Phys. B247 (1984) 373

[31] J. Ellis, K. Enqvist, D.V. Nanopoulos and K. Tamvakis, Phys. Lett. 155B (1985) 381; M. Drees, Phys. Lett. 158B (1985) 409

[32] R. Barbieri and L. Maiani, Nucl. Phys. B243 (1984) 429

[33] H. Komatsu and J. Kubo, Phys. Lett. 157B (1985) 90

[34] E. Ros, 13th International Winter Meeting on Fundamental Physics, Cuenca (Spain), April 1985

[35] H.E. Haber, G.L. Kane, I. Kani and M. Quirós, preprint CERN-TH 4432 and SCIPP 86/51 (1986)

[36] G.L. Kane and W.B. Rolnick, Nucl. Phys. B217 (1983) 117;

B.A. Cambell, J.A. Scott, and M.K. Sundaresan, Phys. Lett. 126B (1983) 376;

W.B. Rolnick, preprint

[37] H.E. Haber and G.L Kane, Nucl. Phys. B232 (1984) 333

[38] K. Enqvist, A. Masiero and D.V. Nanopoulos, Phys. Lett. 156B (1985) 209

[39] J.F. Gunion and H.E. Haber, Nucl. Phys. B272 (1986) 1 